\title{
An adaptive finite element heterogeneous multiscale method for Stokes flow in porous media
}

\author{
Assyr Abdulle*, Ondrej Budáč ${ }^{*}$
}

\begin{abstract}
A finite element heterogeneous multiscale method is proposed for solving the Stokes problem in porous media. The method is based on the coupling of an effective Darcy equation on a macroscopic mesh, with unknown permeabilities recovered from micro finite element calculations for Stokes problems on sampling domains centered at quadrature points in each macro element. The numerical method accounts for non-periodic microscopic geometry that can be obtained from a smooth deformation of a reference pore sampling domain. The computational work is nevertheless independent of the smallness of the pore structure. A priori error estimates reveal that the overall accuracy of the numerical scheme is limited by the regularity of the solutions of the Stokes micro problems. This regularity is low for a typical situation of non-convex microscopic pore geometries. We therefore propose an adaptive scheme with micro-macro mesh refinement driven by residual-based indicators that quantify both the macro and micro errors. A posteriori error analysis is derived for the new method. Two and three dimensional numerical experiments confirm the robustness and the accuracy of the adaptive method.
\end{abstract}

Keywords. Stokes flow, Darcy equation, numerical homogenization, a posteriori error estimates, adaptive finite element method

\section{AMS subject classifications. 65N30, 76D07, 74Qxx, 35Q86}

1. Introduction. Fluid flow through porous media is an important process appearing in a wide range of engineering and technical applications. It is present in the modeling of subsurface contamination and filtration, textile properties, biomedical materials, or natural reservoirs $[30,50,55,56]$. The length-scale of a porous structure is usually much smaller than the computational domain of interest. Standard numerical methods relying on discretization of the porous domain, such as the finite element method (FEM), need to resolve the finest scale of the geometry, which is denoted by $\varepsilon$ in what follows. Such techniques often lead to numerical problems of prohibitive size and computational cost.

In practical applications one is often interested in macroscopic quantities such as bulk properties of the fluid flow. Mathematical models describing such macroscopic quantities are based on averaging techniques such as homogenization. The derivation of effective equations of flow in porous media can be traced back to Darcy [28]. Rigorous homogenization theory of Stokes flow in periodic porous media appeared first in [44] with a proof of convergence by Tartar [47]. This proof was generalized by Allaire [10] to allow for connected solid porous structures in three dimensions. The effective pressure is given by an elliptic (Darcy) equation which contains the effective permeability tensor that depends on the pore geometry and can be computed using the so-called Stokes micro problems. The homogenization theory was further expanded by introducing correctors and $\varepsilon$-dependent error estimates [34] (see also [21]) and to random stochastically homogeneous media [15].

Numerical multiscale algorithms that approximate effective Stokes (or Navier-Stokes) flow in porous media usually rely on a Darcy macro problem. There are numerous works that address extraction of the effective permeability of porous media from the pore geometry of a small representative volume element. Stokes micro problems are then solved on these local pore geometries. We mention explicit analytic results for simple geometries [46], applications to textile modeling [55], and validation of predicted permeability using micro-tomography [36].

\footnotetext{
${ }^{2}$ ANMC, Mathematics Section, École Polytechnique Fédérale de Lausanne, CH-1015 Lausanne, Switzerland; \{assyr.adulle,ondrej.budac\}@epfl.ch.
} 
Some authors assume that the pore structure varies slowly, leading to definitions of slowlyvarying [21] or locally periodic porous media [22,51]. The multiscale FEM described in [20] assumes that the Stokes micro problems can be obtained from a reference periodic domain by a known smooth map and numerically computes micro problems with varying accuracy on nested grids of points. This method relies on high regularity of the Stokes problems that excludes re-entrant corners in micro domains. The two-scale finite element method proposed in [45] was used to compare the multiscale numerical solution of a Darcy equation with a fully resolved fine scale pressure, yielding excellent agreement. A priori analysis or convergence rates of this method were not discussed. The multiscale approach presented in [12] uses control volume method to discretize the Darcy macro equation. The reduced regularity of non-convex micro domains is taken into account and an appropriate estimate of the micro error is derived. The homogenized solution is assumed to have $H^{2}$ regularity.

In this paper we propose an adaptive multiscale method for Stokes flow in porous media. Our method is based on the coupling of an adaptive FEM for elliptic problem with unknown permeability recovered form adaptive FEMs for Stokes problems in microscopic domains. Its construction is obtained as follows. A standard finite element method is used to discretize the Darcy's law on a macroscopic mesh whose size is independent (and can be much larger than) $\varepsilon$. An approximation of the effective permeability is recovered from FE solutions of Stokes problems on micro domains that are centered at quadrature points of the macro elements. We consider stable mixed FE pairs for solution of the Stokes problem as the Taylor-Hood FE [48] or the MINI FE [13]. We then derive a fully discrete a priori error analysis based on [1].

To overcome suboptimal a priori convergence rates caused by non-convexity of the macro and micro domains we propose an adaptive approach. Unlike the adaptive finite element heterogeneous multiscale method (FE-HMM) for elliptic equations $[2,6,40]$ that uses a priori error estimates to control the errors committed in micro problems, we derive a residual-based error estimator that combines the macroscopic residuals and microscopic residuals. We derive an a posteriori upper and lower bound for the error, and we show that our numerical method is both efficient and reliable. This is confirmed in a series of numerical experiments including three-dimensional problems with a non-periodic pore structure.

In this paper we also discuss ellipticity and boundedness of the effective permeability obtained from the velocity fields of Stokes problems on local pore geometries. The well-posedness of the effective Darcy problem is thus closely related to the variation of the pore geometry which we investigate in detail by providing practical criteria to characterize media for which such well-posedness can be established. This is also important to characterize the constants entering in the a posteriori estimates of our numerical method as they also depend on the micro pore geometries.

The organization of the paper is as follows. In Section 2 we review the definition of periodic porous media, define locally periodic porous media, recall the homogenization theory, define the effective problem and investigate its well-posedness. We then define the numerical method in Section 3 and derive a priori error estimates in Section 4. In Section 5 we derive a posteriori upper and lower bound for the numerical method and in Section 6 we describe an adaptive algorithm based on macro and micro residuals obtained from Darcy and Stokes problems. Finally, in Section 7 we present a series of numerical experiments for two and three dimensional problems with non-perioidc porous media that corroborate the optimal performance and robustness of the adaptive multiscale method.

1.1. Notation. Let $C$ denote a generic constant whose value can change at any occurrence but it depends only on explicitly indicated quantities. We consider a domain $\Omega \subset \mathbb{R}^{d}$, $d \in \mathbb{N}$ and the usual Lebesgue space $L^{p}(\Omega)$ and Sobolev space $W^{k, p}(\Omega)$ equipped with the usual norms $\|\cdot\|_{L^{p}(\Omega)}$ and $\|\cdot\|_{W^{k, p}(\Omega)}$. On the factor space $L^{2}(\Omega) / R$, we define $\|q\|_{L^{2}(\Omega) / R}=$ $\inf _{s \in \mathbb{R}}\|q+s\|_{L^{2}(\Omega)}$. For $p=2$ we apply the Hilbert space notation $H^{k}(\Omega)$ and $H_{0}^{1}(\Omega)$ and define the seminorm $|q|_{H^{1}(\Omega)}=\left(\sum_{i=1}^{d}\left\|\partial_{i} q\right\|_{L^{2}(\Omega)}^{2}\right)^{1 / 2}$. The standard scalar product on $L^{2}(\Omega)$ is denoted by $(\cdot, \cdot)_{L^{2}(\Omega)}$. Given a matrix $A \in \mathbb{R}^{d \times d}$ with entries $A_{i j}$, we denote its Frobenius norm by $\|A\|_{\mathrm{F}}=\left(\sum_{i, j=1}^{d} A_{i j}^{2}\right)^{1 / 2}$. Given a vector $\xi \in \mathbb{R}^{d}$ with entries $\xi_{i}$, we define $|\xi|=\left(\sum_{i=1}^{d} \xi_{i}^{2}\right)^{1 / 2}$. Let $\delta_{i j}$ denote the Kronecker delta.

2. Stokes flow in porous media and homogenization. In this section we briefly describe the homogenization of Stokes flow leading to a macroscopic Darcy equation. These 
results were pioneered by Sánchez-Palencia and Tartar [44,47] and refined and extended by many authors (see $[10,35]$ and references therein). We then define locally periodic porous media, state the homogenized model problem, and investigate its well-posedness.

2.1. Periodic porous media. Let $\Omega \subset \mathbb{R}^{d}$ be a bounded, connected, polygonal domain, where $d \in\{2,3\}$. We denote by $Y$ the $d$-dimensional open unit cube $(-1 / 2,1 / 2)^{d}$. Let $Y_{\mathrm{S}} \subset \bar{Y}$ and set $Y_{\mathrm{F}}=Y \backslash Y_{\mathrm{S}}$. Here and subsequently, the subscripts $\mathrm{F}$ and $\mathrm{S}$ stand for the fluid and solid parts of the medium, respectively. For any $r>0$, we denote the $r$-periodic extension of a set $X \subset \mathbb{R}^{d}$ by $E_{r}(X)=\cup_{m \in\left(r / 2+r \mathbb{Z}^{d}\right)}(m+X)$. Let $\varepsilon>0$ and define the periodic porous medium $\Omega_{\varepsilon} \subset \Omega$ by (see Figure 1 )

$$
\Omega_{\varepsilon}=\Omega \backslash\left(\varepsilon E_{1}\left(Y_{\mathrm{S}}\right)\right)
$$

Remark 1. Although $\Omega$ is connected, the definition (1) can lead to $\Omega_{\varepsilon}$ with small parts near the boundary of $\partial \Omega$ that are disconnected from the main body. In what follows, we will neglect these small parts and assume (without changing notation) that $\Omega_{\varepsilon}$ is connected. Moreover, we observe that $\partial \Omega_{\varepsilon}$ does not necessarily have Lipschitz boundary. See Figure 1(c).

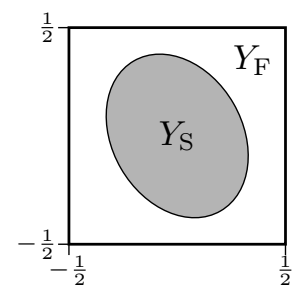

(a)

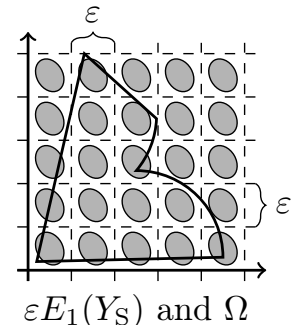

(b)

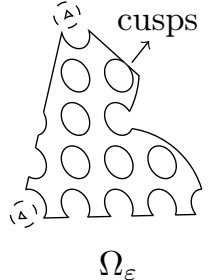

(c)

Figure 1: A pore geometry (a), global pore structure and $\Omega(\mathrm{b})$, and $\Omega_{\varepsilon}$ given by (1) with two cusps and encircled disconnected parts (c).

2.2. Homogenization of Stokes flow. Consider the incompressible Stokes problem in $\Omega_{\varepsilon}$ with the velocity field $\mathbf{u}^{\varepsilon}$, pressure $p^{\varepsilon}$, and force field $\mathbf{f}$, given by

$$
\begin{aligned}
-\Delta \mathbf{u}^{\varepsilon}+\nabla p^{\varepsilon}=\mathbf{f} & \text { in } \Omega_{\varepsilon}, \\
\operatorname{div} \mathbf{u}^{\varepsilon}=0 & \text { in } \Omega_{\varepsilon}, \\
\mathbf{u}^{\varepsilon}=0 & \text { on } \partial \Omega_{\varepsilon} .
\end{aligned}
$$

Homogenization theory examines the limit behavior of the solutions of $(2)$ for $\varepsilon \rightarrow 0^{+}$. Appropriate assumptions on the solid and fluid domains are needed to study these limits (see [10]).

\section{Assumption (H):}

- the set $Y_{\mathrm{S}}$ is closed in $\bar{Y}$, and both $Y_{\mathrm{S}}$ and $Y_{\mathrm{F}}$ have positive measure,

- the sets $\mathbb{R}^{d} \backslash E_{1}\left(Y_{\mathrm{S}}\right)$ and $E_{1}\left(Y_{\mathrm{S}}\right)$ have locally Lipschitz boundaries and are locally located on one side of their boundaries,

- the sets $\mathbb{R}^{d} \backslash E_{1}\left(Y_{\mathrm{S}}\right)$ and $Y_{\mathrm{F}}$ are connected and $Y_{\mathrm{F}}$ has locally Lipschitz boundary.

The analysis proceeds by extending the solution $\mathbf{u}^{\varepsilon}, p^{\varepsilon}$, defined in $\Omega_{\varepsilon}$, to $\mathbf{U}^{\varepsilon}$, $P^{\varepsilon}$, defined in $\Omega$, as described in Remark 2. Assuming $(\mathbf{H})$ it was proved in [10] that $\mathbf{U}^{\varepsilon} / \varepsilon^{2} \rightarrow \mathbf{u}^{0}$ weakly in $L^{2}(\Omega)^{d}$ and $P^{\varepsilon} \rightarrow p^{0}$ strongly in $L_{\text {loc }}^{2}(\Omega) / \mathbb{R}$, where $p^{0}$ is the homogenized pressure given as the solution to the elliptic system

$$
\begin{array}{ll}
\nabla \cdot a^{0}\left(\mathbf{f}-\nabla p^{0}\right)=0 & \text { in } \Omega, \\
a^{0}\left(\mathbf{f}-\nabla p^{0}\right) \cdot \mathbf{n}=0 & \text { on } \partial \Omega .
\end{array}
$$

The so-called homogenized velocity satisfies $\mathbf{u}^{0}=a^{0}\left(\mathbf{f}-\nabla p^{0}\right)$ and the homogenized permeability tensor $a^{0}$ is a $d \times d$ matrix defined by

$$
a^{0}=\int_{Y_{\mathrm{F}}}\left[\mathbf{u}^{1}, \ldots, \mathbf{u}^{d}\right] \mathrm{d} y,
$$


where $\mathbf{u}^{i}(i \in\{1, \ldots, d\})$ is the solution of the following Stokes problem: Find $\mathbf{u}^{i}, p^{i}$ such that

$$
\begin{aligned}
& -\Delta \mathbf{u}^{i}+\nabla p^{i}=\mathbf{e}^{i} \quad \text { in } Y_{\mathrm{F}}, \quad \quad \mathbf{u}^{i}=0 \quad \text { on } \partial E_{1}\left(Y_{\mathrm{S}}\right), \\
& \operatorname{div} \mathbf{u}^{i}=0 \quad \text { in } Y_{\mathrm{F}}, \quad \mathbf{u}^{i} \text { and } p^{i} \quad \text { are } Y \text {-periodic, }
\end{aligned}
$$

where $\mathbf{e}^{i}$ is the $i$-th canonical basis vector in $\mathbb{R}^{d}$.

Remark 2. Suitable extensions of $\mathbf{u}^{\varepsilon}, p^{\varepsilon}$ to $\mathbf{U}^{\varepsilon}, P^{\varepsilon}$ are defined explicitly in [10] as follows. The extension of the velocity is given by $\mathbf{U}^{\varepsilon}(x)=\mathbf{u}^{\varepsilon}(x)$ for $x \in \Omega_{\varepsilon}$ and $\mathbf{U}^{\varepsilon}(x)=0$ for $x \in \Omega \backslash \Omega_{\varepsilon}$. For the pressure, we set $P^{\varepsilon}(x)=p^{\varepsilon}(x)$ for $x \in \Omega_{\varepsilon}$ and for each $z \in\left(1 / 2+\mathbb{Z}^{d}\right)$ with the property $\varepsilon(z+Y) \subset \Omega$, we define $P^{\varepsilon}(x)$ for $x \in\left(\Omega \backslash \Omega_{\varepsilon}\right) \cap \varepsilon(z+Y)$ as the average of $p^{\varepsilon}(w)$ over $w \in \Omega_{\varepsilon} \cap \varepsilon(z+Y)$. Elsewhere in $\Omega \backslash \Omega_{\varepsilon}$, we set $P^{\varepsilon}(x)=0$.

A strong convergence result in the $L^{2}$-norm was derived in [11]. If

$$
\overline{\mathbf{u}}^{0}(x, x / \varepsilon)=\sum_{i=1}^{d} \mathbf{u}^{i}(x / \varepsilon)\left(\mathbf{f}_{i}-\partial_{i} p^{0}\right), \quad \text { then } \quad\left\|\mathbf{U}^{\varepsilon} / \varepsilon^{2}-\overline{\mathbf{u}}^{0}(x, x / \varepsilon)\right\|_{L^{2}(\Omega)} \rightarrow 0 .
$$

Furthermore, under more restrictive conditions on the regularity of $\Omega$ and $Y_{\mathrm{F}}$, an additional corrector $\overline{\mathbf{u}}^{1}$ was defined in [34] allowing for convergence rates (in terms of $\varepsilon$ )

$$
\begin{aligned}
\left\|\mathbf{U}^{\varepsilon} / \varepsilon^{2}-\overline{\mathbf{u}}^{0}(x, x / \varepsilon)-\varepsilon \overline{\mathbf{u}}^{1}(x, x / \varepsilon)\right\|_{H(\Omega, \mathrm{div})} & \leq C \varepsilon^{1 / 6}, \\
\left\|P^{\varepsilon}-p^{0}\right\|_{L^{2}(\Omega) / \mathbb{R}} & \leq C \varepsilon^{1 / 6},
\end{aligned}
$$

where $C$ does not depend on $\varepsilon$.

2.3. Locally periodic porous media. We generalize the definition of periodic porous media from Section 2.1 similarly to [21]. Assume that a reference porous geometry $\left(Y_{\mathrm{S}}, Y_{\mathrm{F}}\right)$ is given, satisfying Assumption (H). Let $\varphi(x, \cdot): \bar{Y} \rightarrow \bar{Y}$ be a homeomorphism for every $x \in \bar{\Omega}$ and assume that $\left.\varphi(x, \cdot)\right|_{\partial Y}$ is an identity. For $\varepsilon \geq 0$ define

$$
\Omega_{\varepsilon}=\Omega \backslash \bigcup_{x \in\left(1 / 2+\mathbb{Z}^{d}\right)} \varepsilon\left(x+\varphi\left(x, Y_{\mathrm{S}}\right)\right) .
$$

Following Remark 1, we assume that $\Omega_{\varepsilon}$ is connected. For any $x \in \Omega$ we define the local pore geometry by $Y_{\mathrm{S}}^{x}=\varphi\left(x, Y_{\mathrm{S}}\right)$ and $Y_{\mathrm{F}}^{x}=Y \backslash Y_{\mathrm{S}}^{x}$. We observe that if $\varphi(x, y) \equiv y$, then we obtain the definition (1).

Example 3. Consider the polynomial $P(c, t)=4(1-c) t^{3}+c t$ that induces a homeomorphism from the closed interval $[-1 / 2,1 / 2]$ to itself for $c \in(0,3 / 2)$. For $d=2$ and $i \in\{1,2\}$ define $\varphi_{i}(x, y)=P\left(5 x_{i}^{2}+0.1, y_{i}\right)$. Finally, we set $\varepsilon=1 / 8$, solid geometry $Y_{\mathrm{S}}=[-1 / 4,1 / 4]^{2}$, and $\Omega=(-0.45,0.45)^{2} \backslash[0,1]^{2}$, as illustrated in Figure 2 .

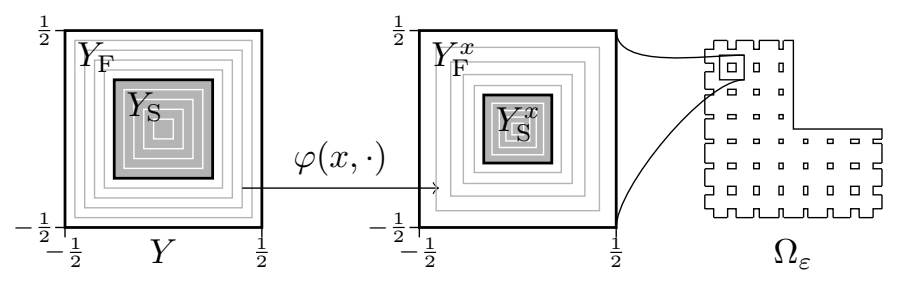

(a)

(b)

(c)

Figure 2: A reference pore structure (a), its image after applying $\varphi(x, \cdot)(\mathrm{b})$, and the locally periodic porous medium $\Omega_{\varepsilon}$ (c) from Example 3.

Remark 4. A level set definition of locally periodic media was described in [22,51]. Given $S: \Omega \times \mathbb{R}^{d} \rightarrow \mathbb{R}$ that is $Y$-periodic in its second variable, we set $\Omega_{\varepsilon}=\{x \in \Omega \mid S(x, x / \varepsilon)>0\}$. For any $x \in \Omega$ we then define $Y_{\mathrm{S}}^{x}=\{y \in \bar{Y} \mid S(x, y) \leq 0\}$ and $Y_{\mathrm{F}}^{x}=Y \backslash Y_{\mathrm{S}}^{x}$.

Remark 5. To allow for some intuitive and simple locally periodic porous media (see the porous medium $C$ in Section 7), we propose a generalization of the presented definition. We let $\varphi: \Omega \times \bar{Y} \times \mathbb{R}_{0}^{+} \rightarrow \bar{Y}$ and use $\varphi\left(x, Y_{\mathrm{S}}, \varepsilon\right)$ in the definition (6). The local geometries are then defined as $Y_{\mathrm{S}}^{x}=\varphi\left(x, Y_{\mathrm{S}}, 0\right)$ and $Y_{\mathrm{F}}^{x}=Y \backslash Y_{\mathrm{S}}^{x}$. Naturally, we assume that $\varphi\left(x, Y_{\mathrm{S}}, \varepsilon\right) \rightarrow$ $\varphi\left(x, Y_{\mathrm{S}}, 0\right)$ for $\varepsilon \rightarrow 0^{+}$. 
2.4. Formal homogenization and the model problem. Following the homogenized problem in periodic porous media $(3),(5),(8)$, we define a model problem in the locally periodic setting. The effective pressure equation (3) stays unchanged but we let $a^{0}$ depend on $x$ as follows. For any $x \in \Omega$ and $i \in\{1, \ldots, d\}$ let $\mathbf{u}^{i, x}, p^{i, x}$ be the velocity and pressure solutions of the the Stokes problem

$$
\begin{aligned}
& -\Delta \mathbf{u}^{i, x}+\nabla p^{i, x}=\mathbf{e}^{i} \quad \text { in } Y_{\mathrm{F}}^{x}, \quad \mathbf{u}^{i, x}=0 \quad \text { on } \partial E_{1}\left(Y_{\mathrm{S}}^{x}\right), \\
& \operatorname{div} \mathbf{u}^{i, x}=0 \quad \text { in } Y_{\mathrm{F}}^{x}, \quad \mathbf{u}^{i, x} \text { and } p^{i, x} \quad \text { are } Y \text {-periodic. }
\end{aligned}
$$

Then set

$$
a^{0}(x)=\int_{Y_{\mathrm{F}}^{x}}\left[\mathbf{u}^{1, x}, \ldots, \mathbf{u}^{d, x}\right] \mathrm{d} y .
$$

Variational formulation. Let $\mathbf{f} \in L^{2}(\Omega)$ and define

$$
\begin{gathered}
W\left(Y_{\mathrm{F}}\right)=\left\{\mathbf{v} \in H^{1}\left(Y_{\mathrm{F}}\right)^{d} ; \mathbf{v}=0 \text { on } \partial E_{1}\left(Y_{\mathrm{S}}\right), \mathbf{v} \text { is } Y \text {-periodic }\right\} \\
a(\mathbf{u}, \mathbf{v})=\sum_{i=1}^{d}\left(\nabla \mathbf{u}_{i}, \nabla \mathbf{v}_{i}\right)_{L^{2}\left(Y_{\mathrm{F}}^{x}\right)^{d}}, \quad \text { and } \quad b(\mathbf{v}, q)=-(q, \operatorname{div} \mathbf{v})_{L^{2}\left(Y_{\mathrm{F}}^{x}\right)}
\end{gathered}
$$

A weak formulation of (7) reads: find $\mathbf{u}^{i, x} \in W\left(Y_{\mathrm{F}}^{x}\right)$ and $p^{i, x} \in L^{2}\left(Y_{\mathrm{F}}^{x}\right) / \mathbb{R}$ such that

$$
\begin{aligned}
a\left(\mathbf{u}^{i, x}, \mathbf{v}\right)+b\left(\mathbf{v}, p^{i, x}\right) & =\left(\mathbf{e}^{i}, \mathbf{v}\right)_{L^{2}\left(Y_{\mathrm{F}}^{x}\right)} & & \forall \mathbf{v} \in W\left(Y_{\mathrm{F}}^{x}\right), \\
b\left(\mathbf{u}^{i, x}, q\right) & =0 & & \forall q \in L^{2}\left(Y_{\mathrm{F}}^{x}\right) / \mathbb{R} .
\end{aligned}
$$

We next recall the weak formulation of $(3)$. Find $p^{0} \in H^{1}(\Omega) / \mathbb{R}$ such that

$$
\begin{gathered}
B_{0}\left(p^{0}, q\right)=L_{0}(q) \quad \forall q \in H^{1}(\Omega) / \mathbb{R} \\
\text { where } \quad B_{0}(p, q)=\int_{\Omega} a^{0} \nabla p \cdot \nabla q \mathrm{~d} x \quad \text { and } \quad L_{0}(q)=\int_{\Omega} a^{0} \mathbf{f} \cdot \nabla q \mathrm{~d} x .
\end{gathered}
$$

Remark 6. The Stokes system (9) can be reformulated by excluding the pressure: find $\mathbf{u}^{i, x} \in V\left(Y_{\mathrm{F}}\right)$ such that

$$
\begin{aligned}
& a\left(\mathbf{u}^{i, x}, \mathbf{v}\right)=\left(\mathbf{e}^{i}, \mathbf{v}\right)_{L^{2}\left(Y_{\mathrm{F}}^{x}\right)} \quad \forall \mathbf{v} \in V\left(Y_{\mathrm{F}}^{x}\right), \\
& \text { where } \quad V\left(Y_{\mathrm{F}}^{x}\right)=\left\{\mathbf{v} \in W\left(Y_{\mathrm{F}}^{x}\right) ; \operatorname{div} \mathbf{v}=0 \text { in } Y_{\mathrm{F}}^{x}\right\} \text {. }
\end{aligned}
$$

Velocity solutions defined by (12) and (9) are identical, see for example [17].

Let us note that the continuous dependence of $a^{0}(x)$ with respect to $x$ can be deduced from the regularity of the map $\varphi(x, y)$. Assuming sufficient smoothness of $\varphi(x, y): \bar{\Omega} \times \bar{Y} \rightarrow \bar{Y}$, the Stokes micro problems (7) can be pulled back to the domain $Y_{\mathrm{F}}$ and we can use continuous dependence of coefficients on $x$ to show that $a^{0} \in C^{0}(\Omega)^{d \times d}$.

2.5. Well-posedness of the model problem. Assuming that $\partial Y_{\mathrm{F}}^{x}$ is piecewise Lipschitz, there is a well-known theory $[33,49]$ that ensures the existence and uniqueness of a weak solution to the problem (9). Consequently, the tensor $a^{0}: \Omega \rightarrow \mathbb{R}^{d \times d}$ is defined uniquely via (8). If $a^{0}$ is uniformly elliptic and bounded, i.e., there is $\Lambda \in \mathbb{R}$ such that $\left\|a^{0}(x)\right\|_{\mathrm{F}} \leq \Lambda$ for a.e. $x \in \Omega$ and there is $\lambda>0$ such that $a^{0}(x) \xi \cdot \xi \geq \lambda|\xi|^{2}$ for each $\xi \in \mathbb{R}^{d}$ and a.e. $x \in \Omega$, then the problem (10) has a unique solution by the classical Lax-Milgram theorem.

It is shown in [44] that $a^{0}$ is elliptic in periodic porous media. However, for locally periodic porous media, this proof does not guarantee uniform ellipticity and boundedness of $a^{0}(x)$. This question turned out to be difficult for arbitrary geometry of the pore structure and is examined in the next subsections.

2.5.1. Uniform boundedness of $a^{0}(x)$. Assume that there is $\alpha_{0} \in \mathbb{R}$ such that, independently of $x \in \Omega$, the following Poincaré-Friedrichs inequality is valid: $\|\mathbf{v}\|_{L^{2}\left(Y_{\mathrm{F}}^{x}\right)} \leq$ $\alpha_{0}|\mathbf{v}|_{H^{1}\left(Y_{\mathrm{F}}^{x}\right.}$ for every $\mathbf{v} \in W\left(Y_{\mathrm{F}}^{x}\right)$. Using the standard estimate of a solution to the Stokes equation (see [33] or [42, Thm. 15.4]) on $\mathbf{u}^{i, x}$, we obtain

$$
\left\|\mathbf{u}^{i, x}\right\|_{L^{2}\left(Y_{\mathrm{F}}^{x}\right)} \leq \alpha_{0}\left|\mathbf{u}^{i, x}\right|_{H^{1}\left(Y_{\mathrm{F}}^{x}\right)} \leq \alpha_{0}\left\|\mathbf{e}^{i}\right\|_{L^{2}\left(Y_{\mathrm{F}}^{x}\right)}<\left|Y_{\mathrm{F}}^{x}\right|^{1 / 2} \alpha_{0}
$$


Further, the Cauchy-Schwarz inequality yields

$$
\left\|a^{0}(x)\right\|_{\mathrm{F}}^{2}=\sum_{i, j=1}^{d}\left(\int_{Y_{\mathrm{F}}^{x}} \mathbf{u}_{j}^{i, x} \mathrm{~d} y\right)^{2} \leq\left|Y_{\mathrm{F}}^{x}\right| \sum_{i=1}^{d}\left\|\mathbf{u}^{i, x}\right\|_{L^{2}\left(Y_{\mathrm{F}}^{x}\right)}^{2} \leq d\left|Y_{\mathrm{F}}^{x}\right|^{2} \alpha_{0}^{2} \leq d \alpha_{0}^{2} .
$$

Hence, $a^{0}(x)$ is uniformly bounded for $x \in \Omega$.

2.5.2. Uniform ellipticity of $a^{0}(x)$. In the first step, we follow the well-known result [44, Ch. 7, Prop. 2.2] and apply it to a non-constant tensor $a^{0}(x)$. Let $i, j \in\{1, \ldots, d\}$ and take $\mathbf{v}=\mathbf{u}^{j, x}$ in (12) to obtain $a\left(\mathbf{u}^{i, x}, \mathbf{u}^{j, x}\right)=\left(\mathbf{e}^{i}, \mathbf{u}^{j, x}\right)_{L^{2}\left(Y_{\mathrm{F}}^{x}\right)}$. Then (8) gives $a_{i j}^{0}(x)=a\left(\mathbf{u}^{i, x}, \mathbf{u}^{j, x}\right)$ which implies the symmetry of $a^{0}(x)$. Define $\mathbf{u}^{\xi, x}=\sum_{i=1}^{d} \xi_{i} \mathbf{u}^{i, x}$ for any $\xi \in \mathbb{R}^{d}$. We have

$$
a^{0}(x) \xi \cdot \xi=\sum_{i, j=1}^{d} \xi_{i} \xi_{j} a\left(\mathbf{u}^{i, x}, \mathbf{u}^{j, x}\right)=\left|\mathbf{u}^{\xi, x}\right|_{H^{1}\left(Y_{\mathrm{F}}^{x}\right)}^{2} \geq 0
$$

To prove that $a^{0}(x)$ is indeed positive definite, we take functions $\mathbf{v}^{i, x} \in V\left(Y_{\mathrm{F}}^{x}\right)$ for $i \in\{1, \ldots, d\}$ with $\left(\mathbf{e}^{j}, \mathbf{v}^{i, x}\right)_{L^{2}\left(Y_{\mathrm{F}}^{x}\right)}=\delta_{i j} \xi_{i}$ for every $j \in\{1, \ldots, d\}$. Then, $a\left(\mathbf{u}^{\xi, x}, \mathbf{v}^{i, x}\right)=\xi_{i}^{2}$, which gives $\mathbf{u}^{\xi, x} \not \equiv 0$ for $\xi \neq 0$, implying a strict inequality in (15) for any $\xi \in \mathbb{R}^{d}, \xi \neq 0$.

The above argument shows the ellipticity of $a^{0}$ but not the uniform ellipticity that we discuss now.

Lemma 7. We have $a^{0}(x) \xi \cdot \xi \geq(\xi, \mathbf{v})_{L^{2}\left(Y_{F}^{x}\right)}^{2} /|\mathbf{v}|_{H^{1}\left(Y_{F}^{x}\right)}^{2}$ for any $\xi \in \mathbb{R}^{d}$ and $\mathbf{v} \in V\left(Y_{F}^{x}\right), \mathbf{v} \not \equiv 0$.

Proof. The equation (12) and the continuity of $a(\cdot, \cdot)$ give

$$
\left|\mathbf{u}^{\xi, x}\right|_{H^{1}\left(Y_{\mathrm{F}}^{x}\right)}|\mathbf{v}|_{H^{1}\left(Y_{\mathrm{F}}^{x}\right)} \geq a\left(\mathbf{u}^{\xi, x}, \mathbf{v}\right)=\sum_{i=1}^{d} \xi_{i} a\left(\mathbf{u}^{i, x}, \mathbf{v}\right)=(\xi, \mathbf{v})_{L^{2}\left(Y_{\mathrm{F}}^{x}\right)} .
$$

The result follows by using (16) to provide a lower bound for $\left|\mathbf{u}^{\xi, x}\right|_{H^{1}\left(Y_{\mathrm{F}}^{x}\right)}$ in (15).

Lemma 8. Let $\nu=\left(\nu_{1}, \ldots, \nu_{d}\right)^{T} \in \mathbb{R}^{d}$. Suppose that for every $i \in\{1, \ldots, d\}$ and $x \in \Omega$ there $i s \mathbf{v}^{i, x} \in V\left(Y_{F}^{x}\right)$ such that $\left|\mathbf{v}^{i, x}\right|_{H^{1}\left(Y_{F}^{x}\right)} \leq \nu_{i}$ and $\left(\mathbf{e}^{j}, \mathbf{v}^{i, x}\right)_{L^{2}\left(Y_{F}^{x}\right)}=\delta_{i j}$ for every $j \in\{1, \ldots, d\}$. Then, $a^{0}(x) \xi \cdot \xi \geq|\nu|^{-2}|\xi|^{2}$ for each $\xi \in \mathbb{R}^{d}$ and $x \in \Omega$.

Proof. Define $\mathbf{v}^{\xi, x}=\sum_{i=1}^{d} \xi_{i} \mathbf{v}^{i, x}$. Notice that $\left(\xi, \mathbf{v}^{\xi, x}\right)_{L^{2}\left(Y_{\mathrm{F}}^{x}\right)}=|\xi|^{2}$ while the triangle and the Cauchy-Schwarz inequality give $\left|\mathbf{v}^{\xi, x}\right|_{H^{1}\left(Y_{\mathrm{F}}^{x}\right)} \leq|\xi||\nu|$. The result follows by setting $\mathbf{v}=\mathbf{v}^{\xi}$ in Lemma 7.

We observe that the representation

$$
(\xi, \mathbf{v})_{L^{2}(\Theta)}=\int_{\partial \Theta}(\xi \cdot y)(\mathbf{v} \cdot \mathbf{n}) \mathrm{d} s
$$

holds for a bounded domain $\Theta \subset \mathbb{R}^{d}$ and a function $\mathbf{v} \in H^{1}(\Theta)^{d}$ with $\operatorname{div} \mathbf{v}=0$. Indeed, using integration by parts we have

$$
\begin{aligned}
\left(\mathbf{e}^{i}, \mathbf{v}\right)_{L^{2}(\Theta)} & =\int_{\partial \Theta} y_{i} \mathbf{v}_{i} \mathbf{n}_{i} \mathrm{~d} s-\int_{\Theta} y_{i} \frac{\partial \mathbf{v}_{i}}{\partial y_{i}} \mathrm{~d} y=\int_{\partial \Theta} y_{i} \mathbf{v}_{i} \mathbf{n}_{i} \mathrm{~d} s+\sum_{j \neq i} \int_{\Theta} y_{i} \frac{\partial \mathbf{v}_{j}}{\partial y_{j}} \mathrm{~d} y \\
& =\int_{\partial \Theta} y_{i} \mathbf{v}_{i} \mathbf{n}_{i} \mathrm{~d} s+\sum_{j \neq i} \int_{\partial \Theta} y_{i} \mathbf{v}_{j} \mathbf{n}_{j} \mathrm{~d} y=\int_{\partial \Theta} y_{i}(\mathbf{v} \cdot \mathbf{n}) \mathrm{d} s=\int_{\partial \Theta}\left(\mathbf{e}^{i} \cdot y\right)(\mathbf{v} \cdot \mathbf{n}) \mathrm{d} s
\end{aligned}
$$

for any $i \in\{1, \ldots, d\}$. The result (17) follows by linearity.

For some pore geometries, Lemma 8 can directly be used to derive (explicit) uniform bounds for the ellipticity of the tensor $a^{0}(x)$. For example, when the fluid part $Y_{\mathrm{F}}^{x}$ contains straight cylindrical subset as described below. We have then an explicit construction for the test functions $\mathbf{v}^{i, x}$ to be used in Lemma 8 using the Poiseuille flow and the uniform bound for the ellipticity of the tensor $a^{0}(x)$ is then explicitly dependent on the radius of the cylinders. 


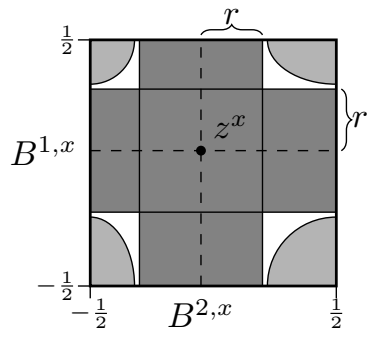

Figure 3: Straight cylindrical subsets of $Y_{\mathrm{F}}^{x}$ from Example 9.

Example 9 (pore geometries $Y_{\mathrm{F}}^{x}$ containing straight cylindrical subsets). Let $r>0, z^{x} \in Y$, and suppose that (see Figure 3)

$$
B^{i, x}=\left\{y \in Y ; r^{2}-\sum_{j \neq i}\left(y_{j}-z_{j}^{x}\right)^{2} \geq 0\right\} \subset Y_{\mathrm{F}}^{x} .
$$

Then $a^{0}(x)$ is uniformly elliptic with $\lambda=r^{3} / 3$ for $d=2$ and $\lambda=\pi r^{4} / 24$ for $d=3$.

This can indeed easily be seen. Let us use a Poiseuille parabolic flow as test functions

$$
\mathbf{v}^{i, x}(y)=C\left(r^{2}-\sum_{j \neq i}\left(y_{j}-z_{j}^{x}\right)^{2}\right) \cdot \begin{cases}\mathbf{e}^{i} & \text { for } y \in B^{i, x} \\ 0 & \text { for } y \in Y_{\mathrm{F}}^{x}-B^{i, x}\end{cases}
$$

It is clear that $\mathbf{v}^{i, x} \in V\left(Y_{\mathrm{F}}^{x}\right)$. Notice that the constant $C$ can be set such that $\left(\mathbf{e}^{j}, \mathbf{v}^{i, x}\right)_{L^{2}\left(Y_{\mathrm{F}}^{x}\right)}=$ $\delta_{i j}$. An explicit computation then allows to estimate $\left|\mathbf{v}^{i, x}\right|_{H^{1}\left(Y_{\mathrm{F}}^{x}\right)} \leq \nu_{i}$ for $i \in\{1, \ldots, d\}$. Using Lemma 8 we conclude the proof.

Example 10 (pore geometries $Y_{\mathrm{F}}^{x}$ containing curved cylindrical subsets). Let $r>0$ and for $i \in\{1, \ldots, d\}$ consider $B^{i, x}$, as defined in (18), but without assuming $B^{i, x} \subset Y_{\mathrm{F}}^{x}$. Suppose that $\Phi^{i, x}: B^{i, x} \rightarrow \tilde{B}^{i, x}=\Phi^{i, x}\left(B^{i, x}\right) \subset Y_{\mathrm{F}}^{x}$ is a $C^{1}$-diffeomorphism such that

(a) $\Phi^{i, x}(y)=y$ for all $y \in \partial B^{i, x} \cap \partial Y$,

(b) $\partial_{j} \Phi_{i}^{i, x}(y)=0$ for $y \in B^{i, x}$ and $j \neq i$,

(c) there is $L>0$ independent of $x \in \Omega$ and $i \in\{1, \ldots, d\}$ such that the $C^{1}$-norms of $\Phi^{i, x}$ and $\left(\Phi^{i, x}\right)^{-1}$ are bounded by $L$.

Then $a^{0}(x)$ is uniformly elliptic with $\lambda$ depending only on $r, d$, and $L$.
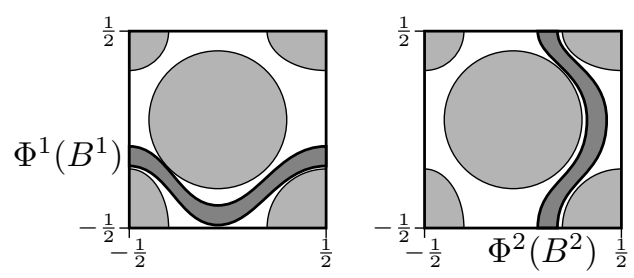

Figure 4: Curved cylindrical subsets from Example 10.

An illustration of a possible geometry that fulfills the above assumptions is given in Figure 4 . To construct suitable test functions to be used in Lemma 8, we take the functions $\mathbf{v}^{i, x}$ from (19) and define $\tilde{\mathbf{v}}^{i, x} \in H^{1}\left(Y_{\mathrm{F}}^{x}\right)$ by $\tilde{\mathbf{v}}^{i, x}(y)=\mathbf{v}^{i, x}\left(\left(\Phi^{i, x}\right)^{-1}(y)\right)$ for $y \in \tilde{B}^{i, x}$ and $\tilde{\mathbf{v}}^{i, x}(y)=0$ elsewhere in $Y_{\mathrm{F}}^{x}$. The assumption (a) implies $\tilde{\mathbf{v}}^{i} \in W\left(Y_{\mathrm{F}}^{x}\right)$ and using (b) one can prove $\tilde{\mathbf{v}}^{i, x} \in V\left(Y_{\mathrm{F}}^{x}\right)$. The assumption (a) and the observation (17) lead to $\left(\mathbf{e}^{j}, \tilde{\mathbf{v}}^{i, x}\right)=\left(\mathbf{e}^{j}, \mathbf{v}^{i, x}\right)=\delta_{i j}$ for all $j \in\{1, \ldots, d\}$. We observe that $\left|\tilde{\mathbf{v}}^{i, x}\right|_{1}$ can be bounded by an explicit constant depending on $r, d$, and $L$. We then conclude by applying Lemma 8 with test functions $\tilde{\mathbf{v}}^{i, x}$.

Remark 11. The assumption (b) from Example 10 is needed to show that $\tilde{\mathbf{v}}^{i, x}$ is divergencefree. For $d=2$, this assumption can be removed provided a higher regularity of $\Phi^{i, x}$. Indeed, we take $q^{i, x} \in H^{2}(Y)$ such that $\mathbf{v}^{i, x}=\left[\partial_{2} q^{i, x},-\partial_{1} q^{i, x}\right]$, where $\mathbf{v}^{i, x}$ is defined in (19). Then, we can define $\tilde{q}^{i, x}=q^{i, x}\left(\left(\Phi^{i, x}\right)^{-1}(y)\right)$ and $\tilde{\mathbf{v}}^{i, x}(y)=\left[\partial_{2} \tilde{q}^{i, x},-\partial_{1} \tilde{q}^{i, x}\right]$. Notice that we need $\Phi^{i, x}$ to be a $C^{2}$-diffeomorphism. 
The next step is to simplify Lemma 8 such that the test functions $\mathbf{v}^{i, x}$ do not have to be divergence-free. We start by introducing the inf-sup constant of $b(\cdot, \cdot)$ in Definition 12 .

Definition 12. For any $x \in \Omega$ let $\beta_{0}^{x}>0$ be the constant from the following inf-sup condition: for every $q \in L^{2}\left(Y_{F}^{x}\right) / \mathbb{R}$ there is $\mathbf{v} \in H_{0}^{1}\left(Y_{F}^{x}\right)^{d}$ such that $b(\mathbf{v}, q) \geq \beta_{0}^{x}\|q\|_{L^{2}\left(Y_{F}^{x}\right) / \mathbb{R}}|\mathbf{v}|_{H^{1}\left(Y_{F}^{x}\right)}$.

In what follows, we will assume a uniform inf-sup constant for all domains $Y_{\mathrm{F}}^{x}$ with $x \in \Omega$, i.e., $\beta_{0}^{x} \geq \beta$ for every $x \in \Omega$. Such results can be obtained for a large class of geometries than can be expressed as a union of star-shape domains with respect to open balls [31, Chap. III.3]. We note however that for domains with thin channels or large aspect ratio, $\beta$ degenerate with increasing aspect ratio [29].

Lemma 13. Let $\eta=\left(\eta_{1}, \ldots, \eta_{d}\right)^{T} \in \mathbb{R}^{d}$. Suppose that for any $x \in \Omega$ and $i \in\{1, \ldots, d\}$ there is $\mathbf{w}^{i, x} \in W\left(Y_{F}^{x}\right)$ such that $\left|\mathbf{w}^{i, x}\right|_{H^{1}\left(Y_{F}^{x}\right)} \leq \eta_{i}$ and $\int_{\partial Y_{F}^{x}} y_{j}\left(\mathbf{w}^{i, x} \cdot \mathbf{n}\right) \mathrm{d} y=\delta_{i j}$ for every $j \in\{1, \ldots, d\}$. Further, suppose that there exists $\beta>0$ such that $\beta_{0}^{x} \geq \beta$ for every $x \in \Omega$. Then $a^{0}(x) \xi \cdot \xi \geq \lambda|\xi|^{2}$ for each $\xi \in \mathbb{R}^{d}$ and $x \in \Omega$ with $\lambda$ depending only on $\beta$, $\eta$, and the dimension $d$.

Proof. Let $\tilde{\mathbf{v}}^{i, x} \in H_{0}^{1}\left(Y_{\mathrm{F}}^{x}\right)^{d}$ and $\tilde{p}^{i, x} \in L^{2}\left(Y_{\mathrm{F}}^{x}\right) / \mathbb{R}$ be given by the Stokes problem

$$
\begin{aligned}
a\left(\tilde{\mathbf{v}}^{i, x}, \mathbf{w}\right)+b\left(\mathbf{w}, \tilde{p}^{i, x}\right) & =-a\left(\mathbf{w}^{i, x}, \mathbf{w}\right) & & \forall \mathbf{w} \in H_{0}^{1}\left(Y_{\mathrm{F}}^{x}\right)^{d}, \\
b\left(\tilde{\mathbf{v}}^{i, x}, q\right) & =-b\left(\mathbf{w}^{i, x}, q\right) & & \forall q \in L^{2}\left(Y_{\mathrm{F}}^{x}\right) / \mathbb{R} .
\end{aligned}
$$

Define $\mathbf{v}^{i, x}=\tilde{\mathbf{v}}^{i, x}+\mathbf{w}^{i, x}$. One can show that $\mathbf{v}^{i, x}$ is divergence-free, yielding $\mathbf{v}^{i, x} \in V\left(Y_{\mathrm{F}}^{x}\right)$. Standard Stokes estimates $[33,42]$ give

$$
\left|\mathbf{v}^{i, x}\right|_{H^{1}\left(Y_{\mathrm{F}}^{x}\right)} \leq 2\left(1+\sqrt{d} / \beta_{0}^{x}\right)\left|\mathbf{w}^{i, x}\right|_{H^{1}\left(Y_{\mathrm{F}}^{x}\right)} \leq 2(1+\sqrt{d} / \beta) \eta_{i}=: \nu_{i}
$$

Further, using (17) and the condition $\mathbf{v}^{i, x}=\mathbf{w}^{i, x}$ on $\partial Y_{\mathrm{F}}^{x}$ yield $\left(\mathbf{e}^{j}, \mathbf{v}^{i, x}\right)_{L^{2}\left(Y_{\mathrm{F}}^{x}\right)}=\delta_{i j}$ for any $j \in\{1, \ldots, d\}$. Using Lemma 8 gives $\lambda=|\nu|^{-2}$, with $\nu=\left(\nu_{1}, \ldots, \nu_{d}\right)^{T}$.

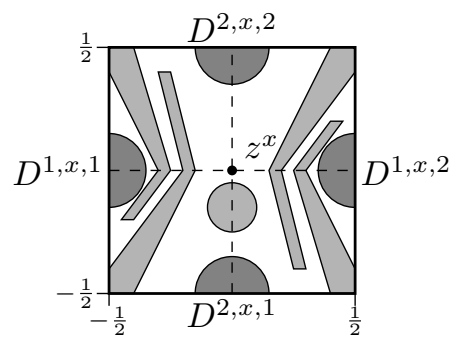

Figure 5: Half-balls from the construction of test functions in Example 14.

We now apply Lemma 13 to derive uniform bounds for the ellipticity of the tensor $a^{0}(x)$ in a general situation.

Example 14. Let $r>0$ and $z^{x} \in Y$ be such that the half-balls

$$
D^{i, x, k}=\{y \in Y ; \underbrace{r^{2}-\left(y_{i}+(-1)^{k} / 2\right)^{2}-\sum_{j \neq i}\left(y_{j}-z_{j}^{x}\right)^{2}}_{=: g^{i, x, k}(y)} \geq 0\}
$$

(see Figure 5) satisfy $D^{i, x, k} \subset Y_{\mathrm{F}}^{x}$ for $i \in\{1, \ldots, d\}$ and $k \in\{1,2\}$. Then $a^{0}(x)$ is uniformly elliptic with $\lambda$ depending only on $r$ and $\beta$. Indeed, define

$$
\mathbf{w}^{i, x}(y)=C g^{i, x, k}(y) \cdot \begin{cases}\mathbf{e}^{i} & \text { for } y \in D^{i, x, k}, k \in\{1,2\} \\ 0 & \text { otherwise }\end{cases}
$$

where $C$ is a constant depending on $r$ that assures $\int_{\partial Y_{\mathrm{F}}^{x}} x_{j}\left(\mathbf{w}^{i, x} \cdot \mathbf{n}\right) \mathrm{d} y=\delta_{i j}$ for every $j \in$ $\{1, \ldots, d\}$. Using test functions $\mathbf{w}^{i, x}$ in Lemma 13 concludes the proof. 
3. DS-FE-HMM for the Stokes Problem. In this section we define the numerical multiscale method for the Stokes problem in porous media with an acronym $D S-F E$ HMM (Darcy-Stokes finite element heterogeneous multiscale method). Since the effective equation (10) is elliptic, we can use the strategy developed in [1] for the macro solver. The coupling with micro problems, however, differs from the FE-HMM as we now have to solve micro Stokes problems. There we use mixed finite elements.

3.1. Definition of the method. Let $\varepsilon>0$ and assume that $\Omega$ and $\Omega_{\varepsilon}$ are connected bounded polygonal domains in $\mathbb{R}^{d}$ with $\Omega_{\varepsilon} \subset \Omega$. Let $\left\{\mathcal{T}_{H}\right\}$ be a family of conformal, shaperegular triangulations of $\Omega$ parametrized by the mesh size $H=\max _{K \in \mathcal{T}_{H}} H_{K}$, where $H_{K}=$ $\operatorname{diam}(K)$.

Macro FE space and quadrature formulas. We consider the macro FE space

$$
S_{H}^{l}(\Omega)=\left\{q^{H} \in H^{1}(\Omega) ;\left.q^{H}\right|_{K} \in \mathcal{P}^{l}(K), \forall K \in \mathcal{T}_{H}\right\},
$$

where $\mathcal{P}^{l}(K)$ is the space of polynomials on $K$ of degree $l \in \mathbb{N}$. For the sake of simplicity of notation, we indicate the dependence of spaces $W_{h}\left(Y_{\mathrm{F}}^{x, \delta}\right)$ and $L_{h}\left(Y_{\mathrm{F}}^{x, \delta}\right)$ on the micro triangulation $\mathcal{T}_{h}^{x}$ only by the subscript $h$. For each element $K \in \mathcal{T}_{H}$ we consider an affine mapping $F_{K}$ such that $K=F_{K}(\hat{K})$, where $\hat{K}$ is the simplicial reference element. Let $J \in \mathbb{N}$ and $\left\{\hat{x}_{j}, \hat{\omega}_{j}\right\}_{j=1}^{J}$ be a given quadrature formula on $\hat{K}$ with positive weights and $\hat{x}_{j} \in \hat{K}$. The transformation $F_{K}$ induces a quadrature formula on $K$ with integration points $x_{K_{j}}=F_{K}\left(\hat{x}_{j}\right)$ and weights $\omega_{K_{j}}=\hat{\omega}_{j}\left|\operatorname{det}\left(\partial F_{K}\right)\right|$. Denote

$$
Q^{K}=\left\{x_{K_{j}}\right\}_{j=1}^{J} \quad \text { and } \quad Q^{H}=\cup_{K \in \mathcal{T}_{H}} Q^{K},
$$

where we notice that $Q^{K} \subset K$ and $Q^{H} \subset \Omega$. Denote the family of all quadrature points for $H>0$ by $\left\{Q^{H}\right\}$. Since exact integration will be replaced by a quadrature formula, we need the following assumption to recover well-posedness of the numerical method and guarantee the optimal order of accuracy, see [24, Chap. 4.1].

Assumption (Q). For $m=\max (2 l-2, l)$ and any $\hat{q}(\hat{x}) \in \mathcal{P}^{m}(\hat{K})$ we assume

$$
\int_{\hat{K}} \hat{q}(\hat{x}) \mathrm{d} \hat{x}=\sum_{j=1}^{J} \hat{\omega}_{j} \hat{q}\left(\hat{x}_{j}\right) .
$$

The assumption (Q) is valid for example if $J=1, \omega_{K_{1}}=|K|$, and $x_{K_{1}}$ is the barycenter of $K$. See $[26,27]$ and the references therein for further examples of quadrature formulas.

Micro FE spaces. Let $\delta \geq \varepsilon$. For each $x \in\left\{Q^{H}\right\}$ we define the local geometry snapshot

$$
Y_{\mathrm{S}}^{x, \delta}=\left(\left(\left(\mathbb{R}^{d}-\Omega_{\varepsilon}\right) \cap(x+\delta \bar{Y})\right)-x\right) / \varepsilon, \quad Y_{\mathrm{F}}^{x, \delta}=((\delta / \varepsilon) Y) \backslash Y_{\mathrm{S}}^{x, \delta} .
$$

For any $x \in\left\{Q^{H}\right\}$, we assume that $\left\{\mathcal{T}_{h}^{x}\right\}_{h}$ is a family of conformal, shape-regular triangulations of $Y_{\mathrm{F}}^{x, \delta}$ parametrized by the mesh size $h=\max _{T \in \mathcal{T}_{h}^{x}} h_{T}$, where $h_{T}=\operatorname{diam}(T)$. The shaperegularity constants are assumed to be the same for each $x \in\left\{Q^{H}\right\}$ and $\delta \geq \varepsilon$. We consider two standard stable pairs of micro velocity and pressure elements (see [17]): the Taylor-Hood $\mathbb{P}_{k+1} / \mathbb{P}_{k} \mathrm{FE}$ for $k \geq 1$ and the MINI FE (see Remark 15). We consider two different boundary conditions $(\mathrm{BC})$ on the micro scale: periodic and Neumann. The pressure FE space is given by

$$
L_{h}\left(Y_{\mathrm{F}}^{x, \delta}\right)= \begin{cases}\left\{q \in S_{h}^{k}\left(Y_{\mathrm{F}}^{x, \delta}\right) ; q \text { is }(\delta / \varepsilon) Y \text {-periodic }\right\} & \text { for periodic BC, } \\ S_{h}^{k}\left(Y_{\mathrm{F}}^{x, \delta}\right) & \text { for Neumann BC, }\end{cases}
$$

The velocity FE space is given by

$$
W_{h}\left(Y_{\mathrm{F}}^{x, \delta}\right)=\bar{W}\left(Y_{\mathrm{F}}^{x, \delta}\right) \cap S_{h}^{k+1}\left(Y_{\mathrm{F}}^{x, \delta}\right)^{d},
$$

where

$$
\bar{W}\left(Y_{\mathrm{F}}^{x, \delta}\right)=\left\{\begin{array}{cl}
\left\{\mathbf{v} \in H^{1}\left(Y_{\mathrm{F}}^{x, \delta}\right)^{d} ; \mathbf{v}=0 \text { on } \partial E_{\delta / \varepsilon}\left(Y_{\mathrm{S}}^{x, \delta}\right),\right. & \\
\mathbf{v} \text { is }(\delta / \varepsilon) Y \text {-periodic }\} & \text { for periodic BC } \\
\left\{\mathbf{v} \in H^{1}\left(Y_{\mathrm{F}}^{x, \delta}\right)^{d} ; \mathbf{v}=0 \text { on } \partial Y_{\mathrm{S}}^{x, \delta}\right\} & \text { for Neumann BC. }
\end{array}\right.
$$


For the sake of simplicity of notation, we indicate the dependence of spaces $W_{h}\left(Y_{\mathrm{F}}^{x, \delta}\right)$ and $L_{h}\left(Y_{\mathrm{F}}^{x, \delta}\right)$ on the micro triangulation $\mathcal{T}_{h}^{x}$ only by the subscript $h$.

Remark 15. To use MINI finite elements, we take (23) with $k=1$ and

$$
W_{h}\left(Y_{\mathrm{F}}^{x, \delta}\right)=\bar{W}\left(Y_{\mathrm{F}}^{x, \delta}\right) \cap\left(S_{h}^{1}\left(Y_{\mathrm{F}}^{x, \delta}\right)^{d} \oplus \mathcal{B}\left(Y_{\mathrm{F}}^{x, \delta}, \mathcal{T}_{h}^{x}\right)^{d}\right),
$$

where $\mathcal{B}$ is the bubble space $\mathcal{B}\left(Y_{\mathrm{F}}^{x, \delta}, \mathcal{T}_{h}^{x}\right)=\left\{q \in S_{h}^{d+1}\left(Y_{\mathrm{F}}^{x, \delta}\right) ; q=0\right.$ on $\left.\partial K, \forall K \in \mathcal{T}_{h}^{x}\right\}$.

In case of periodic boundary conditions, we assume that the micro meshes $\mathcal{T}_{h}^{x}$ are conformal over periodic boundaries and periodicity can be thus enforced strongly (see Figure 6(c)).

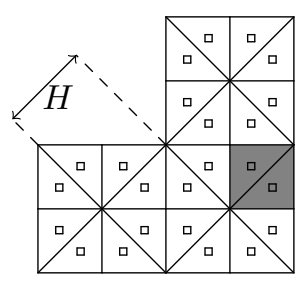

(a)

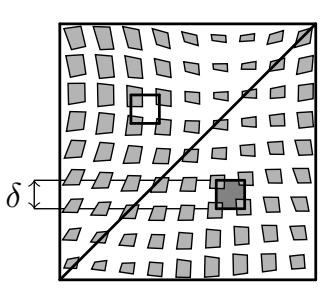

(b)

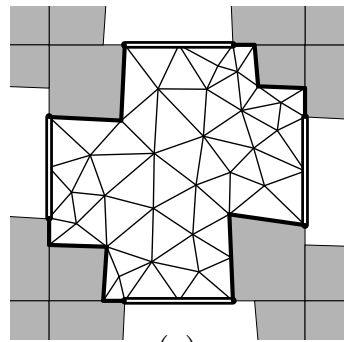

(c)

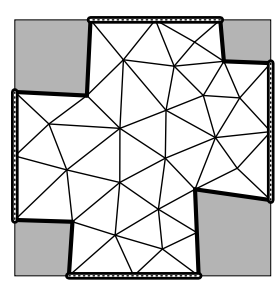

(d)

Figure 6: DS-FE-HMM with $\mathbb{P}_{1}$ macro elements $(l=1)$ : (a) $\mathcal{T}_{H}$; (b) zoom on $\Omega_{\varepsilon}$ in the two highlighted macro elements; (c) $\mathcal{T}_{h}^{x}$ in $Y_{\mathrm{F}}^{x, \delta}$ with periodic coupling (periodic: Il, Dirichlet: I); (d) $\mathcal{T}_{h}^{x}$ in $Y_{\mathrm{F}}^{x, \delta}$ with Neumann coupling (Neumann: 目, Dirichlet: I).

Coupling macro and micro methods (DS-FE-HMM). The coupled Darcy-Stokes finite element heterogeneous multiscale method (DS-FE-HMM) is defined as follows. Find $p^{H} \in$ $S_{H}^{l}(\Omega) / \mathbb{R}$ such that

$$
B_{H}\left(p^{H}, q^{H}\right)=L_{H}\left(q^{H}\right) \quad \forall q^{H} \in S_{H}^{l}(\Omega) / \mathbb{R},
$$

where the discrete macro bilinear form and right-hand side corresponding to (10), (11) are

$$
\begin{aligned}
B_{H}\left(p^{H}, q^{H}\right) & =\sum_{K \in \mathcal{T}_{H}} \sum_{j=1}^{J} \omega_{K_{j}} a^{h}\left(x_{K_{j}}\right) \nabla p^{H}\left(x_{K_{j}}\right) \cdot \nabla q^{H}\left(x_{K_{j}}\right), \\
L_{H}\left(q^{H}\right) & =\sum_{K \in \mathcal{T}_{H}} \sum_{j=1}^{J} \omega_{K_{j}} a^{h}\left(x_{K_{j}}\right) \mathbf{f}^{H}\left(x_{K_{j}}\right) \cdot \nabla q^{H}\left(x_{K_{j}}\right),
\end{aligned}
$$

Here, $\mathbf{f}^{H} \in \tilde{S}_{H}^{l-1}(\Omega)^{d}$ is an appropriate interpolation of the force field $\mathbf{f} \in L^{2}(\Omega)^{d}$, where

$$
\tilde{S}_{H}^{l-1}(\Omega)=\left\{q^{H} \in L^{2}(\Omega) ;\left.q^{H}\right|_{K} \in \mathcal{P}^{l-1}(K), \forall K \in \mathcal{T}_{H}\right\},
$$

and $a^{h}\left(x_{K_{j}}\right)$ is a numerical approximation of the tensor $a^{0}\left(x_{K_{j}}\right)$ computed by the micro Stokes problems: for each $i \in\{1, \ldots, d\}$ and quadrature point $x \in Q^{H}$ find $\mathbf{u}^{i, x, h} \in W_{h}\left(Y_{\mathrm{F}}^{x, \delta}\right)$ and $p^{i, x, h} \in L_{h}\left(Y_{\mathrm{F}}^{x, \delta}\right) / \mathbb{R}$ such that

$$
\begin{aligned}
a\left(\mathbf{u}^{i, x, h}, \mathbf{v}\right)+b\left(\mathbf{v}, p^{i, x, h}\right) & =\left(\mathbf{e}^{i}, \mathbf{v}\right)_{L^{2}\left(Y_{\mathrm{F}}^{x, \delta}\right)} & & \forall \mathbf{v} \in W_{h}\left(Y_{\mathrm{F}}^{x, \delta}\right) \\
b\left(\mathbf{u}^{i, x, h}, q\right) & =0 & & \forall q \in L_{h}\left(Y_{\mathrm{F}}^{x, \delta}\right) / \mathbb{R}
\end{aligned}
$$

and set

$$
a^{h}(x)=\frac{\varepsilon^{d}}{\delta^{d}} \int_{Y_{\mathrm{F}}^{x, \delta}}\left[\mathbf{u}^{1, x, h}, \ldots, \mathbf{u}^{d, x, h}\right] \mathrm{d} y .
$$

Remark 16. Neumann boundary conditions in the micro problems are especially useful when the periodically extended fluid part $\mathbb{R}^{d} \backslash E_{\delta / \varepsilon}\left(Y_{\mathrm{S}}^{x, \delta}\right)$ is not connected (see for example Figure 7 (right)). There, periodic boundary conditions would yield $a^{h}(x)=0$. 
Computational cost. Denote the number of macroscopic degrees of freedom by $N_{\text {mac }}$ and the (average) number of microscopic degrees of freedom by $N_{\text {mic }}$. If the time cost of solving one (micro or macro) problem is assumed to be linear in the degrees of freedom, the total cost of the DS-FE-HMM method is $\mathcal{O}\left(N_{\text {mic }} N_{\text {mac }}\right)$, which does not depend on the pore size $\varepsilon$. Indeed, in general $\delta \geq \varepsilon$ is of size comparable to $\varepsilon$ and $\delta=\varepsilon$ can be chosen for periodic problems.

Velocity reconstruction. We reconstruct a discontinuous velocity field using piecewise approximation of $a^{h}\left(\mathbf{f}^{H}-\nabla p^{H}\right)$ by interpolation from quadrature points. In addition to the assumption (Q) we assume that the number of quadrature nodes $J$ is minimal, i.e., $J=$ $\left(\begin{array}{c}l+d-1 \\ d\end{array}\right)$. Given a macro element $K \in \mathcal{T}_{H}$ (recall the definition $(21)$ ) and a function $q: Q^{K} \rightarrow \mathbb{R}$, there is a unique interpolant $\Pi(q) \in \mathcal{P}^{l-1}(K)$ such that $\Pi(q)(x)=q(x)$ for every quadrature point $x \in Q^{K}$ (see [37, Prop. 50], [7]). Therefore, for any tensor $a: Q^{H} \rightarrow \mathbb{R}^{d \times d}$, there is a unique operator $\Pi_{a}$ that maps $\tilde{S}_{H}^{l-1}(\Omega)$ to itself and satisfies

$$
\Pi_{a}(\mathbf{v})(x)=a(x) \mathbf{v}(x), \quad \forall x \in Q^{H} .
$$

We define the DS-FE-HMM velocity reconstruction by $\mathbf{u}^{H}=\Pi_{a^{h}}\left(\mathbf{f}^{H}-\nabla p^{H}\right)$.

Remark 17. Quadrature formulas that satisfy (Q) and $J=\left(\begin{array}{c}l+d-1 \\ d\end{array}\right)$ are known only for $l \leq 3$ in two dimensions and $l \leq 2$ in three dimensions, see [26] and the references therein. If the number of quadrature nodes is not minimal, we can still define $\Pi(q)$ as

$$
\Pi(q)=\underset{r \in \mathcal{P}^{l-1}(K)}{\operatorname{argmin}} \sum_{j=1}^{J} \omega_{K_{j}}\left|q\left(x_{K_{j}}\right)-r\left(x_{K_{j}}\right)\right|^{2} .
$$

We note that the assumption $(\mathbf{Q})$ and definition of $\Pi$ give

$$
\begin{aligned}
B_{H}\left(p^{H}, q^{H}\right) & =\sum_{K \in \mathcal{T}_{H}} \sum_{j=1}^{J} \omega_{K_{j}} a^{h}\left(x_{K_{j}}\right) \nabla p^{H}\left(x_{K_{j}}\right) \cdot \nabla q^{H}\left(x_{K_{j}}\right) \\
& =\sum_{K \in \mathcal{T}_{H}} \sum_{j=1}^{J} \omega_{K_{j}} \Pi_{a^{h}}\left(\nabla p^{H}\right)\left(x_{K_{j}}\right) \cdot \nabla q^{H}\left(x_{K_{j}}\right) \\
& =\sum_{K \in \mathcal{T}_{H}} \int_{K} \Pi_{a^{h}}\left(\nabla p^{H}\right) \cdot \nabla q^{H} \mathrm{~d} x=\int_{\Omega} \Pi_{a^{h}}\left(\nabla p^{H}\right) \cdot \nabla q^{H} \mathrm{~d} x
\end{aligned}
$$

and analogously

$$
L_{H}\left(q^{H}\right)=\sum_{K \in \mathcal{T}_{H}} \int_{K} \Pi_{a^{h}}\left(\mathbf{f}^{H}\right) \cdot \nabla q^{H}=\int_{\Omega} \Pi_{a^{h}}\left(\mathbf{f}^{H}\right) \cdot \nabla q^{H} .
$$

3.2. Well-posedness of the DS-FE-HMM. There is a well-known theory $[13,17,19]$ that guarantees well-posedness of the micro problems (27) with finite element spaces defined in (23) or (24). The well-posedness of the macro problem (25) relies on the Lax-Milgram lemma.

Proposition 18. In addition to Assumption (Q), assume that there exist $\Lambda, \lambda>0$ such that $\left\|a^{h}(x)\right\|_{\mathrm{F}} \leq \Lambda$ and $a^{h}(x) \xi \cdot \xi \geq \lambda|\xi|^{2}$ for all $\xi \in \mathbb{R}^{d}$, quadrature points $x \in Q^{H}$, and $h>0$. Then there is a unique solution $p^{H}$ of (25). Moreover, $\left|p^{H}\right|_{H^{1}(\Omega)} \leq \Lambda / \lambda\left\|\mathbf{f}^{H}\right\|_{L^{2}(\Omega)}$.

Proof. The assumptions imply continuity of $L_{H}(\cdot)$ and $B_{H}(\cdot, \cdot)$ and ellipticity of $B_{H}(\cdot, \cdot)$. Indeed, for any $q^{H}, r^{H} \in S_{H}^{l}(\Omega)$, we have

$$
\begin{aligned}
& B_{H}\left(q^{H}, q^{H}\right) \geq \lambda \sum_{K \in \mathcal{T}_{H}} \sum_{j=1}^{J} \omega_{K_{j}}\left|\nabla q^{H}\left(x_{K_{j}}\right)\right|^{2}=\lambda\left|q^{H}\right|_{H^{1}(\Omega)}^{2}, \\
& B_{H}\left(q^{H}, r^{H}\right) \leq \Lambda\left|q^{H}\right|_{H^{1}(\Omega)}\left|r^{H}\right|_{H^{1}(\Omega)}, \quad L_{H}\left(q^{H}\right) \leq \Lambda\left\|\mathbf{f}^{H}\right\|_{L^{2}(\Omega)}\left|q^{H}\right|_{H^{1}(\Omega)} .
\end{aligned}
$$

The Lax-Milgram lemma concludes the proof. 
We now study the conditions under which $a^{h}(x)$ is uniformly elliptic and bounded. In particular, the dependence of $\Lambda$ and $\lambda$ on the local geometries $Y_{\mathrm{F}}^{x, \delta}$ is discussed.

Let us first examine the case of micro problems (27) being solved exactly in Sobolev spaces. Using here the variant of Stokes problem (12), which excludes pressure, we arrive at the following definition. For any $x \in\left\{Q^{H}\right\}$ and $i \in\{1, \ldots, d\}$, let $\overline{\mathbf{u}}^{i, x} \in \bar{V}\left(Y_{\mathrm{F}}^{x, \delta}\right)$ be the unique solution to the Stokes problem

$$
\begin{gathered}
a\left(\overline{\mathbf{u}}^{i, x}, \overline{\mathbf{v}}\right)=\left(\mathbf{e}^{i}, \overline{\mathbf{v}}\right)_{L^{2}\left(Y_{\mathrm{F}}^{x, \delta}\right)} \quad \forall \overline{\mathbf{v}} \in \bar{V}\left(Y_{\mathrm{F}}^{x, \delta}\right), \\
\text { where } \quad \bar{V}\left(Y_{\mathrm{F}}^{x, \delta}\right)=\left\{\overline{\mathbf{v}} \in \bar{W}\left(Y_{\mathrm{F}}^{x, \delta}\right) ; \operatorname{div} \overline{\mathbf{v}}=0 \text { in } Y_{\mathrm{F}}^{x, \delta}\right\} .
\end{gathered}
$$

We then define

$$
\bar{a}(x)=\frac{\varepsilon^{d}}{\delta^{d}} \int_{Y_{\mathrm{F}}^{x, \delta}}\left[\overline{\mathbf{u}}^{1, x}, \ldots, \overline{\mathbf{u}}^{d, x}\right] \mathrm{d} y .
$$

If we show that $\bar{a}(x)$ is elliptic for every $x \in Q^{H}$, then we can already claim that, for a given macro mesh $\mathcal{T}_{H}$, the tensor $a^{h}(x)$ is elliptic for each $x \in Q^{H}$ provided that $h>0$ is sufficiently small. Indeed, the convergence properties of the stable FE scheme (27) assure that

$$
\lim _{h \rightarrow 0}\left\|a^{h}(x)-\bar{a}(x)\right\|_{\mathrm{F}}=0 \quad \forall x \in Q^{H} .
$$

We thus examine the ellipticity of $\bar{a}(x)$ and then show how a global (independent of $x \in\left\{Q^{H}\right\}$ ) ellipticity constant of $a^{h}(x)$ can be obtained for $h<\hat{h}$, where $\hat{h}>0$ also does not depend on $x \in\left\{Q^{H}\right\}$.

3.2.1. Uniform ellipticity of $\bar{a}(x)$. Independently of the boundary conditions (periodic or Neumann) that are used in the Stokes micro problems (27), we can apply the ellipticity results from Section 2.5.2 on the tensor $\bar{a}(x)$ with very little changes. One has to substitute the spaces $W\left(Y_{\mathrm{F}}^{x}\right)$ and $V\left(Y_{\mathrm{F}}^{x}\right)$ by the spaces $\bar{W}\left(Y_{\mathrm{F}}^{x, \delta}\right)$ and $\bar{V}\left(Y_{\mathrm{F}}^{x, \delta}\right)$, respectively, and apply the scaling factor $(\varepsilon / \delta)^{d}$ appearing in the definition of $\bar{a}(x)$. Analogously to Lemma 7 we get

$$
\bar{a}(x) \xi \cdot \xi \geq(\varepsilon / \delta)^{d}(\xi, \overline{\mathbf{v}})_{L^{2}\left(Y_{\mathrm{F}}^{x, \delta}\right)}^{2} /|\overline{\mathbf{v}}|_{H^{1}\left(Y_{\mathrm{F}}^{x, \delta}\right)}^{2} \quad \forall \xi \in \mathbb{R}^{d}, \quad \forall \overline{\mathbf{v}} \in \bar{V}\left(Y_{\mathrm{F}}^{x, \delta}\right), \quad \overline{\mathbf{v}} \not \equiv 0 .
$$

However, there is an important class of pore geometries $\left(Y_{\mathrm{F}}^{x, \delta}, Y_{\mathrm{S}}^{x, \delta}\right)$ where adapting results from Section 2.5.2 does not yield a desirable estimate, see for example Figure 7 (right). When the fluid part $Y_{\mathrm{F}}^{x, \delta}$ is not connected over opposite edges of $(\delta / \varepsilon) Y$, periodic boundary conditions in micro problems will yield $\overline{\mathbf{u}}^{i, x} \equiv 0$, implying $\bar{a}(x)=0$ and finally $a^{h}(x)=0$. To avoid such scenarios, Neumann boundary conditions can be used. To account for such geometries, we start with a variant of Lemma 8 that shows ellipticity of $\bar{a}(x)$ with weaker assumptions on the scalar products of test functions with $\mathbf{e}^{j}$.
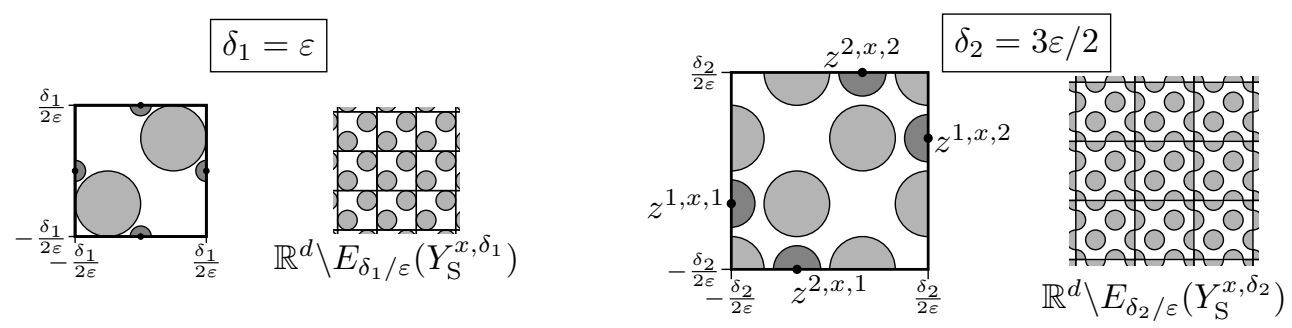

Figure 7: Considering a porous medium with circular solid parts (light gray), we take two pore geometries $\left(Y_{\mathrm{F}}^{x, \delta}, Y_{\mathrm{S}}^{x, \delta}\right)$ of different sizes $\delta_{1}$ (left) and $\delta_{2}$ (right). Each pore geometry is periodically repeated in $\mathbb{R}^{2}$. Half-balls at the boundaries (dark gray) indicate the use of either Example 14 (left) or Example 21 (right).

Lemma 19. Let $L>0$ and $\eta=\left(\eta_{1}, \ldots, \eta_{d}\right)^{T} \in \mathbb{R}^{d}$. Assume that for any $x \in\left\{Q^{H}\right\}$ and $i \in\{1, \ldots, d\}$ there is $\overline{\mathbf{v}}^{i, x} \in \bar{V}\left(Y_{F}^{x, \delta}\right)$ such that $\left|\overline{\mathbf{v}}^{i, x}\right|_{H^{1}\left(Y_{F}^{x, \delta}\right)} \leq \eta_{i}$, and denote $A_{i j}^{x}=$ $\left(\mathbf{e}^{i}, \overline{\mathbf{v}}^{j, x}\right)_{L^{2}\left(Y_{F}^{x, \delta}\right)}$ for any $i, j \in\{1, \ldots, d\}$. Assume that the matrix $A^{x}$ is invertible with $\left\|\left(A^{x}\right)^{-1}\right\|_{F} \leq L$. Then $\bar{a}(x) \xi \cdot \xi \geq \lambda|\xi|^{2}$ for each $\xi \in \mathbb{R}^{d}$ and $x \in\left\{Q^{H}\right\}$ with $\lambda=C L^{2}|\eta|^{-2}$ and $C$ depending only on the dimension $d$. 
Proof. Set $B^{x}=\left(A^{x}\right)^{-1}$ and define $\overline{\mathbf{v}}^{\xi, x}=\sum_{j=1}^{d} \xi_{i} B_{j i}^{x} \overline{\mathbf{v}}^{j, x}$. Notice that $\left(\xi, \overline{\mathbf{v}}^{\xi, x}\right)_{L^{2}\left(Y_{\mathrm{F}}^{x}\right)}=|\xi|^{2}$, while the triangle and the Cauchy-Schwarz inequality give $\left|\overline{\mathbf{v}}^{\xi, x}\right|_{H^{1}\left(Y_{\mathrm{F}}^{x}\right)} \leq C L|\eta||\xi|$, where $C$ depends only on $d$. The result follows by setting $\overline{\mathbf{v}}=\overline{\mathbf{v}}^{\xi}$ in (34).

The previous lemma can be used to obtain generalizations of Example 10, Lemma 13, and Example 14 that allow for non-periodic test functions. As an illustration, we show a simple way to generalize Example 14.

Definition 20. For any quadrature point $x \in\left\{Q^{H}\right\}$ let $\beta_{\delta}^{x}>0$ be the constant from the following inf-sup condition: for every $\bar{q} \in L^{2}\left(Y_{F}^{x, \delta}\right) / \mathbb{R}$ there is $\overline{\mathbf{v}} \in \bar{W}\left(Y_{F}^{x, \delta}\right)$ such that $b(\overline{\mathbf{v}}, \bar{q}) \geq$ $\beta_{\delta}^{x}\|\bar{q}\|_{L^{2}\left(Y_{F}^{x, \delta}\right) / \mathbb{R}}|\overline{\mathbf{v}}|_{H^{1}\left(Y_{F}^{x, \delta}\right)} \cdot$

Example 21. Suppose that the inf-sup constants $\beta_{\delta}^{x}$ have a lower bound $\beta_{\delta}^{x}>\beta_{\delta}>0$ for every quadrature point $x \in\left\{Q^{H}\right\}$. For any $i \in\{1, \ldots, d\}$, any quadrature point $x \in\left\{Q^{H}\right\}$, and any $k \in\{1,2\}$, let $z^{i, x, k} \in \partial Y$ such that $z_{i}^{i, x, k}=(-1)^{k} \delta /(2 \varepsilon)$. Define a matrix $A^{x}$ by $A_{i j}^{x}=z_{j}^{i, x, 2}-z_{j}^{i, x, 1}$ and suppose that there exists $\gamma>0$ such that $\left|\operatorname{det}\left(A^{x}\right)\right| \geq \gamma$. Further, assume that the half-balls

$$
D^{i, x, k}=\left\{y \in Y ; r^{2}-\sum_{j=1}^{d}\left(y_{j}-z_{j}^{i, x, k}\right)^{2} \geq 0\right\}
$$

(see Figure 7 (right)) satisfy $D^{i, x, k} \subset Y_{\mathrm{F}}^{x, \delta}$ for $i \in\{1, \ldots, d\}$ and $k \in\{1,2\}$, where $r>0$ is a given radius. Then $\bar{a}(x)$ is uniformly elliptic with $\lambda$ depending only on $r, \gamma, \beta_{\delta}$, and $\delta / \varepsilon$. Indeed, define

$$
\overline{\mathbf{w}}^{i, x}(y)=C\left(r^{2}-\sum_{j=1}^{d}\left(y_{j}-z_{j}^{i, x, k}\right)^{2}\right) \cdot \begin{cases}\mathbf{e}^{i} & \text { for } y \in D^{i, x, k}, k \in\{1,2\}, \\ 0 & \text { otherwise }\end{cases}
$$

where $C$ is the constant from Example 14, depending only on $r$. Direct integration gives $\int_{\partial Y_{\mathrm{F}}^{x}} y_{j}\left(\overline{\mathbf{w}}^{i, x} \cdot \mathbf{n}\right) \mathrm{d} y=A_{i j}^{x}$ for any $j \in\{1, \ldots, d\}$. Using the auxiliary Stokes problems as in Lemma 13 and then the formula (17), we can find $\overline{\mathbf{v}}^{i, x} \in \bar{V}\left(Y_{\mathrm{F}}^{x, \delta}\right)$ such that $\left(\mathbf{e}^{j}, \overline{\mathbf{v}}^{i, x}\right)=A_{i j}^{x}$ and $\left|\overline{\mathbf{v}}^{i, x}\right|_{H^{1}\left(Y_{\mathrm{F}}^{x, \delta}\right)} \leq C$, where $C$ depends on $r$ and $\beta_{\delta}$. Finally, the assumption on $A^{x}$ (observe also that $\left.\left\|A^{x}\right\|_{\mathrm{F}} \leq(\delta / \varepsilon) d\right)$ gives $\left\|\left(A^{x}\right)^{-1}\right\|_{\mathrm{F}} \leq C$, where $C$ depends only on $\gamma, d$, and $\delta / \varepsilon$. We can conclude by using the test functions $\overline{\mathbf{v}}^{i, x}$ in Lemma 19 .

3.2.2. Uniform boundedness of $a^{h}(x)$. We follow the reasoning from Section 2.5.1. Assume that there is $\alpha \in \mathbb{R}$ such that the Poincaré-Friedrichs inequality $\|\mathbf{v}\|_{L^{2}\left(Y_{\mathrm{F}}^{x, \delta}\right)} \leq$ $\alpha|\mathbf{v}|_{H^{1}\left(Y_{\mathrm{F}}^{x, \delta}\right)}$ is valid for every $\mathbf{v} \in \bar{W}\left(Y_{\mathrm{F}}^{x, \delta}\right)$, independently of $\delta \geq \varepsilon$ and quadrature point $x \in\left\{Q^{H}\right\}$. Following the estimates (13), (14), while using the standard discrete solution stability result (see $[33,42])$ and $W_{h}\left(Y_{\mathrm{F}}^{x, \delta}\right) \subset \bar{W}\left(Y_{\mathrm{F}}^{x, \delta}\right)$, we obtain $\left\|a^{h}(x)\right\|_{\mathrm{F}} \leq \sqrt{d} \alpha$.

3.2.3. Uniform ellipticity of $a^{h}(x)$. Similarly to Section 2.5.2, we derive lower bounds of $a^{h}(x) \xi \cdot \xi$. Using here the variant of Stokes problem (12), which excludes pressure, we can restate (27) as follows. For any $x \in\left\{Q^{H}\right\}$ and $i \in\{1, \ldots, d\}$ find $\mathbf{u}^{i, x, h} \in V_{h}\left(Y_{\mathrm{F}}^{x, \delta}\right)$ such that

$$
a\left(\mathbf{u}^{i, x, h}, \mathbf{v}\right)=\left(\mathbf{e}^{i}, \mathbf{v}^{h}\right)_{L^{2}\left(Y_{\mathrm{F}}^{x, \delta}\right)} \quad \forall \mathbf{v}^{h} \in V_{h}\left(Y_{\mathrm{F}}^{x, \delta}\right),
$$

where

$$
V_{h}\left(Y_{\mathrm{F}}^{x, \delta}\right)=\left\{\mathbf{v}^{h} \in W_{h}\left(Y_{\mathrm{F}}^{x, \delta}\right) ; b\left(\mathbf{v}^{h}, q^{h}\right)=0, \forall q^{h} \in L_{h}\left(Y_{\mathrm{F}}^{x, \delta}\right) / \mathbb{R}\right\} .
$$

As in Section 2.5.2, we obtain $a_{i j}^{h}(x)=(\varepsilon / \delta)^{d} a\left(\mathbf{u}^{i, x, h}, \mathbf{u}^{j, x, h}\right)$, which implies that $a^{h}(x)$ is symmetric. Following Lemma 7 we get

$$
a^{h}(x) \xi \cdot \xi \geq(\varepsilon / \delta)^{d}\left(\xi, \mathbf{v}^{h}\right)_{L^{2}\left(Y_{\mathrm{F}}^{x, \delta}\right)}^{2} /\left|\mathbf{v}^{h}\right|_{H^{1}\left(Y_{\mathrm{F}}^{x, \delta}\right)}^{2} \quad \forall \xi \in \mathbb{R}^{d}, \quad \forall \mathbf{v}^{h} \in V_{h}\left(Y_{\mathrm{F}}^{x, \delta}\right), \mathbf{v}^{h} \not \equiv 0 .
$$

The main idea in what follows is to take, for any $x \in\left\{Q^{H}\right\}$ and any unit vector $\eta \in \mathbb{R}^{d}$, a test function $\overline{\mathbf{v}}^{\eta, x} \in \bar{V}\left(Y_{\mathrm{F}}^{x, \delta}\right)$ that satisfies the property

$$
\left(\eta, \overline{\mathbf{v}}^{\eta, x}\right)_{L^{2}\left(Y_{\mathrm{F}}^{x, \delta}\right)}^{2} \geq \gamma\left|\overline{\mathbf{v}}^{\eta, x}\right|_{H^{1}\left(Y_{\mathrm{F}}^{x, \delta}\right)}^{2}
$$


where $\gamma>0$ is a constant. Notice that $\overline{\mathbf{v}}^{\eta, x}$ is a velocity solution to the Stokes problem: find $\overline{\mathbf{v}} \in \bar{W}\left(Y_{\mathrm{F}}^{x, \delta}\right)$ and $\bar{p} \in L^{2}\left(Y_{\mathrm{F}}^{x, \delta}\right) / \mathbb{R}$ such that

$$
\begin{aligned}
a(\overline{\mathbf{v}}, \overline{\mathbf{w}})+b(\overline{\mathbf{w}}, \bar{p}) & =a\left(\overline{\mathbf{v}}^{\eta, x}, \overline{\mathbf{w}}\right) & & \forall \overline{\mathbf{w}} \in \bar{W}\left(Y_{\mathrm{F}}^{x, \delta}\right), \\
b(\overline{\mathbf{v}}, \bar{q}) & =0 & & \forall \bar{q} \in L^{2}\left(Y_{\mathrm{F}}^{x, \delta}\right) / \mathbb{R} .
\end{aligned}
$$

Let $\mathbf{v}^{\eta, x, h} \in W_{h}\left(Y_{\mathrm{F}}^{x, \delta}\right)$ be a discrete velocity solution of (39) where we replace the continuous spaces $\bar{W}\left(Y_{\mathrm{F}}^{x, \delta}\right)$ and $L^{2}\left(Y_{\mathrm{F}}^{x, \delta}\right) / \mathbb{R}$ by the discrete spaces $W_{h}\left(Y_{\mathrm{F}}^{x, \delta}\right)$ and $L_{h}\left(Y_{\mathrm{F}}^{x, \delta}\right)$, respectively. Standard Stokes estimates (see $[33,49])$ give

$$
\lim _{h \rightarrow 0} \frac{\left|\mathbf{v}^{\eta, x, h}-\overline{\mathbf{v}}^{\eta, x}\right|_{H^{1}\left(Y_{\mathrm{F}}^{x, \delta}\right)}}{\left|\overline{\mathbf{v}}^{\eta, x}\right|_{H^{1}\left(Y_{\mathrm{F}}^{x, \delta}\right)}}=0 .
$$

The following lemma now combines these considerations into an ellipticity result for $a^{h}$.

Lemma 22. Suppose that for each quadrature point $x \in\left\{Q^{H}\right\}$, and unit vector $\eta \in \mathbb{R}^{d}$, there exists $\overline{\mathbf{v}}^{\eta, x} \in \bar{V}\left(Y_{F}^{x, \delta}\right)$ with the property (38), where $\gamma>0$ is a constant and let $\mathbf{v}^{\eta, x, h} \in V_{h}\left(Y_{F}^{x, \delta}\right)$ be defined as described after (39). Assume that there are uniform bounds on the Poicaré-Friedrichs inequalities introduced in Sections 2.5.1 and 3.2.2 denoted by $\alpha_{0}$ and $\alpha$, respectively. Finally, assume that the functions $\mathbf{v}^{\eta, x, h} \in V_{h}\left(Y_{F}^{x, \delta}\right)$ satisfy the limit condition (40) uniformly (with respect to quadrature points $x \in\left\{Q^{H}\right\}$ and $\eta \in \mathbb{R}^{d}$ ). Then there is $\lambda>0$ and $\hat{h}>0$ such that $a^{h}(x) \xi \cdot \xi \geq \lambda|\xi|^{2}$ for all $h>0$ with $h<\hat{h}$, vectors $\xi \in \mathbb{R}^{d}$, and $x \in\left\{Q^{H}\right\}$.

Proof. A simple computation gives

$$
\left|\frac{\left(\eta, \mathbf{v}^{\eta, x, h}\right)_{L^{2}\left(Y_{\mathrm{F}}^{x, \delta}\right)}^{2}}{\left|\mathbf{v}^{\eta, x, h}\right|_{H^{1}\left(Y_{\mathrm{F}}^{x, \delta}\right)}^{2}}-\frac{\left(\eta, \overline{\mathbf{v}}^{\eta, x}\right)_{L^{2}\left(Y_{\mathrm{F}}^{x, \delta}\right)}^{2}}{\left|\overline{\mathbf{v}}^{\eta, x}\right|_{H^{1}\left(Y_{\mathrm{F}}^{x, \delta}\right)}^{2}}\right| \leq C \frac{\left|\mathbf{v}^{\eta, x, h}-\overline{\mathbf{v}}^{\eta, x}\right|_{H^{1}\left(Y_{\mathrm{F}}^{x, \delta}\right)}^{2}}{\left|\overline{\mathbf{v}}^{\eta, x}\right|_{H^{1}\left(Y_{\mathrm{F}}^{x, \delta}\right)}^{2}},
$$

where $C$ depends on $\delta / \varepsilon$ and on the Poincaré-Friedrichs constants $\alpha_{0}$ and $\alpha$. Using the assumption (40) we know that there is $\hat{h}>0$ such that for all $h \leq \hat{h}$ the right-hand side in (41) can be bounded by $\gamma / 2$. That in turn gives $\left(\xi, \mathbf{v}^{\eta, x, h}\right)_{L^{2}\left(Y_{\mathrm{F}}^{x, \delta}\right)}^{2} \geq \gamma / 2\left|\mathbf{v}^{\eta, x, h}\right|_{H^{1}\left(Y_{\mathrm{F}}^{x, \delta}\right)}^{2}$ for all $h<\hat{h}$. The estimate $(37)$ concludes the proof with $\lambda=\gamma(\varepsilon / \delta)^{d} / 2$.

Remark 23. Test functions $\overline{\mathbf{v}}^{\eta, x}$ in Lemma 22 can be constructed in many ways. One can use the test functions from the proofs of coercivity of $\bar{a}$ in Section 3.2.1 or we can take directly $\overline{\mathbf{v}}^{\eta, x}=\sum_{i=1}^{d} \eta_{i} \overline{\mathbf{u}}^{i, x}$, where $\overline{\mathbf{u}}^{i, x}$ is defined in (31). The only additional assumption is the uniformity of the limit behavior (40).

We close this section by a construction of the test functions for Lemma 22 in a specific situation, where the rate of convergence of (40) can be derived explicitly. First, we need a definition of a discrete inf-sup condition.

Definition 24. For any quadrature point $x \in\left\{Q^{H}\right\}$ let $\beta_{\delta}^{x, h}>0$ be the constant from the following inf-sup condition: for every $q^{h} \in L_{h}\left(Y_{F}^{x, \delta}\right) / \mathbb{R}$ there is $\mathbf{v}^{h} \in W_{h}\left(Y_{F}^{x, \delta}\right)$ such that $b\left(\mathbf{v}^{h}, q^{h}\right) \geq \beta_{\delta}^{x, h}\left\|q^{h}\right\|_{L^{2}\left(Y_{F}^{x, \delta}\right) / \mathbb{R}}\left|\mathbf{v}^{h}\right|_{H^{1}\left(Y_{F}^{x, \delta}\right)}$.

For every $x \in\left\{Q^{H}\right\}$ we have continuous $\left(\beta_{\delta}^{x}\right)$ and a numerical $\left(\beta_{\delta}^{x, h}\right)$ inf-sup constants. As in Section 3.2.1, we assume that there is $\beta_{\delta}>0$ such that $\beta_{\delta}^{x} \geq \beta_{\delta}$ for all $x \in \Omega$. Then for stable pairs of finite element spaces in shape-regular meshes (see [17]), we also have a uniform bound for the discrete inf-sup constants, i.e., there is $\beta_{\delta, \text { num }}>0$ such that $\beta_{\delta}^{x, h} \geq \beta_{\delta \text {,num }}$ independently of $x \in\left\{Q^{H}\right\}$ and $h>0$. Recall that the constant of shape-regularity for the families of triangulations $\left\{\mathcal{T}_{h}^{x}\right\}_{h}$ is assumed to be independent of $x \in\left\{Q^{H}\right\}$.

Example 25. Let $M \geq \mu(\delta / \varepsilon)^{d-1}$, where $\mu>0$ is a constant. Assume that there is $r>0$ such that for every quadrature point $x \in\left\{Q^{H}\right\}$ and $m \in\{1, \ldots, M\}$, there is a point $z^{x, m} \in(\delta / \varepsilon) Y$ with the following properties. The cylinders

$$
B^{i, x, m}=\left\{y \in(\delta / \varepsilon) Y: r^{2}-\sum_{j \neq i}\left(y_{j}-z_{j}^{x, m}\right)^{2} \geq 0\right\}
$$


satisfy $B^{i, x, m} \subset Y_{\mathrm{F}}^{x, \delta}$ and if $m \neq n$, then $B^{i, x, m} \cap B^{i, x, n}=\emptyset$ (see Figure 8 ). Then there are test functions $\overline{\mathbf{v}}^{\eta, x}$ satisfying the assumptions of Lemma 22. Indeed, we can define

$$
\overline{\mathbf{v}}^{i, x}(y)=C\left(r^{2}-\sum_{j \neq i}\left(y_{j}-z_{j}^{x, m}\right)^{2}\right)^{2} \cdot \begin{cases}\mathbf{e}^{i} & \text { for } y \in B^{i, x, m}, m \in\{1, \ldots, M\}, \\ 0 & \text { for } y \in Y_{\mathrm{F}}^{x, \delta} \backslash \cup_{m=1}^{M} B^{i, x, m},\end{cases}
$$

where $C$ is the constant from Example 9 depending only on $r$, which then gives $\left(\mathbf{e}^{j}, \overline{\mathbf{v}}^{i, x}\right)=$ $\delta_{i j} M(\delta / \varepsilon)^{d}$. We set $\overline{\mathbf{v}}^{\eta, x}=\sum_{i=1}^{d} \eta_{i} \overline{\mathbf{v}}^{i, x}$ for any unit vector $\eta \in \mathbb{R}^{d}$ and show that these test functions satisfy the conditions of Lemma 22. A direct computation shows that (38) is satisfied with $\gamma=C(\delta / \varepsilon)^{d}$, where $C>0$ depends only on $\mu, r$, and $d$. Notice also (using only (42)) that $\overline{\mathbf{v}}^{\eta, x} \in H^{2}\left(Y_{\mathrm{F}}^{x, \delta}\right)^{d} \cap \bar{V}\left(Y_{\mathrm{F}}^{x, \delta}\right)$ and $\left\|\overline{\mathbf{v}}^{\eta, x}\right\|_{H^{2}\left(Y_{\mathrm{F}}^{x, \delta}\right)}$ can be computed explicitly. Recall that $\mathbf{v}^{\eta, x, h}$ is defined in Lemma 22 as a discrete solution of a Stokes problem where the continuous solution is $\overline{\mathbf{v}}^{\eta, x}$. Using standard approximation results (see $[33,49]$ ) we obtain

$$
\left|\overline{\mathbf{v}}^{\eta, x}-\mathbf{v}^{\eta, x, h}\right|_{H^{1}\left(Y_{\mathrm{F}}^{x, \delta}\right)} \leq C h\left\|\overline{\mathbf{v}}^{\eta, x}\right\|_{H^{2}\left(Y_{\mathrm{F}}^{x, \delta}\right)} \leq C h\left|\overline{\mathbf{v}}^{\eta, x}\right|_{H^{1}\left(Y_{\mathrm{F}}^{x, \delta}\right)},
$$

where $C$ depends on $r, d$, the lower bound on the discrete inf-sup constants $\beta_{\delta \text {,num, micro }}$ FE space, and the shape regularity constant of $\mathcal{T}_{h}^{x}$. The estimate (43) implies a uniform convergence rate in (40). Hence, all the assumptions of Lemma 22 are satisfied and the coercivity constant $\lambda$ only depends on $\mu, r$ and $d$ (and not on $\delta>\varepsilon$ and $x \in\left\{Q^{H}\right\}$ ).

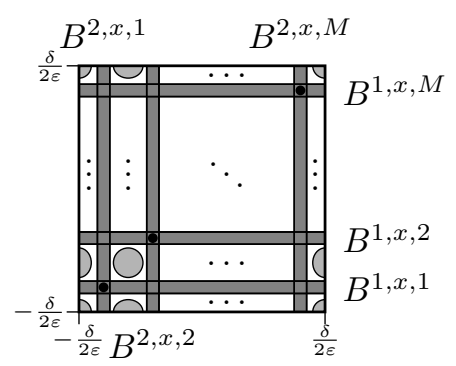

Figure 8: Straight cylindrical subsets of $Y_{\mathrm{F}}^{x, \delta}$ from Example 25. Points $z^{x, m}$ are marked by bullets on the intersections of $B^{1, x, m}$ and $B^{2, x, m}$.

4. A priori error estimates. To estimate the error between the DS-FE-HMM solution $p^{H}$ of (25) and the homogenized solution $p^{0}$ of (46), we follow [2] and decompose the error in three parts, the macro, the micro and the modeling error, as

$$
|\underbrace{p^{0}-p^{H}}_{e}|_{H^{1}(\Omega)} \leq|\underbrace{p^{0}-p^{0, H}}_{e_{\text {mac }}}|_{H^{1}(\Omega)}+|\underbrace{p^{0, H}-\bar{p}^{H}}_{e_{\text {mod }}}|_{H^{1}(\Omega)}+|\underbrace{\bar{p}^{H}-p^{H}}_{e_{\text {mic }}}|_{H^{1}(\Omega)} .
$$

The functions $p^{0, H}, \bar{p}^{H}$, solutions of problems (45), (46), respectively, are FE solutions of a problem similar to (25) but with different effective tensors as described below.

4.1. Preliminaries. We start with the semi-discrete $D S-F E-H M M$. This is a version of the DS-FE-HMM where the micro problems (27) are solved exactly, in Sobolev spaces, yielding micro velocities $\overline{\mathbf{u}}^{i, x} \in \bar{W}\left(Y_{\mathrm{F}}^{x, \delta}\right)$ and tensor $\bar{a}(x)$ as described in (36) and (32), respectively. We search $\bar{p}^{H} \in S_{H}^{l}(\Omega) / \mathbb{R}$ such that

$$
\bar{B}_{H}\left(\bar{p}^{H}, q^{H}\right)=\bar{L}_{H}\left(q^{H}\right) \quad \forall q^{H} \in S_{H}^{l}(\Omega) / \mathbb{R},
$$

where $\bar{B}_{H}, \bar{L}_{H}$ are given by $(26)$ with $a^{h}(x)$ replaced by $\bar{a}(x)$. The reconstructed velocity is then defined as $\overline{\mathbf{u}}^{H}=\Pi_{\bar{a}}\left(\mathbf{f}^{H}-\nabla \bar{p}^{H}\right)$.

We next consider the classical FEM with numerical quadrature for the elliptic macro problem with exact effective tensor. Find $p^{0, H} \in S_{H}^{l}(\Omega) / \mathbb{R}$ such that

$$
B_{0, H}\left(p^{0, H}, q^{H}\right)=L_{0, H}\left(q^{H}\right) \quad \forall q^{H} \in S_{H}^{l}(\Omega) / \mathbb{R},
$$

where where $B_{0, H}, L_{0, H}$ are given by (26) with $a^{h}(x)$ replaced by $a^{0}(x)$. Finally, let $\mathbf{u}^{0, H}=$ $\Pi_{a^{0}}\left(\mathbf{f}^{H}-\nabla p^{0, H}\right)$. 
Remark 26. The well-posedness of the intermediate (in terms of discretness) problems (45) and (46) depends on the properties of the tensors $\bar{a}(x)$ and $a^{0}(x)$, which are examined in Section 3.2 and Section 2.5, respectively.

4.2. Macro error. If Assumption (Q) holds, $p^{0} \in H^{l+1}(K)$, and $a^{0}$ is uniformly elliptic, bounded, and sufficiently smooth, then

$$
\left|p^{0}-p^{0, H}\right|_{H^{1}(\Omega)} \leq C\left(H^{l}+\left\|\mathbf{f}-\mathbf{f}^{H}\right\|_{L^{2}(\Omega)}\right),
$$

where $C$ is independent of $H$ and $\varepsilon$ (see [24, Chap. 4.1]). If $\Omega$ is a convex and $a^{0}$ is sufficiently regular, we have $p^{0} \in H^{2}(\Omega)$. If $\mathbf{f} \in H^{l-1}(\Omega)$, we can bound the term $\left\|\mathbf{f}-\mathbf{f}^{H}\right\|_{L^{2}(\Omega)}$ by $C H^{l}$.

For the velocity field we have the estimate

$$
\left\|\mathbf{u}^{0}-\mathbf{u}^{0, H}\right\|_{L^{2}(\Omega)} \leq C\left(\left|p^{0}-p^{0, H}\right|_{H^{1}(\Omega)}+\left\|a^{0}\left(\mathbf{f}^{H}-\nabla p^{0, H}\right)-\Pi_{a^{0}}\left(\mathbf{f}^{H}-\nabla p^{0, H}\right)\right\|_{L^{2}(\Omega)}\right) .
$$

We can decompose the second term of the previous estimate further to $\left\|a^{0} \mathbf{f}^{H}-\Pi_{a^{0}}\left(\mathbf{f}^{H}\right)\right\|_{L^{2}(\Omega)}$ and $\left\|a^{0} p^{H}-\Pi_{a^{0}}\left(p^{H}\right)\right\|_{L^{2}(\Omega)}$. These terms vanish if we assume that $a^{0}(x)$ is constant within each element $K \in \mathcal{T}_{H}$. In a general situation, these terms can be bounded by $C H^{l}$, if higher derivatives of $\mathbf{f}^{H}$ and $p^{H}$ are bounded.

4.3. Modeling error. Using the ellipticity and boundedness of the bilinear form $\bar{B}_{H}(\cdot, \cdot)$ (see Remark 26) and recalling that $e_{\bmod }=p^{0, H}-\bar{p}^{H}$, we obtain

$$
\begin{aligned}
\left|p^{0, H}-\bar{p}^{H}\right|_{H^{1}(\Omega)} & \leq C \frac{\left|\bar{B}_{H}\left(p^{0, H}, e_{\mathrm{mod}}\right)-\bar{B}_{H}\left(\bar{p}^{H}, e_{\mathrm{mod}}\right)\right|}{\left|e_{\mathrm{mod}}\right| H^{1}(\Omega)} \\
& \leq C \frac{\left|\bar{B}_{H}\left(p^{0, H}, e_{\mathrm{mod}}\right)-B_{0, H}\left(p^{0, H}, e_{\mathrm{mod}}\right)\right|+\left|\bar{L}_{H}\left(e_{\mathrm{mod}}\right)-L_{0, H}\left(e_{\mathrm{mod}}\right)\right|}{\left|e_{\mathrm{mod}}\right| H^{1}(\Omega)} \\
& \leq \sup _{q^{H} \in S_{H}^{l}(\Omega)} C \frac{\left|\bar{B}_{H}\left(p^{0, H}, q^{H}\right)-B_{0, H}\left(p^{0, H}, q^{H}\right)\right|+\left|\bar{L}_{H}\left(q^{H}\right)-L_{0, H}\left(q^{H}\right)\right|}{\left|q^{H}\right|_{H^{1}(\Omega)}} \\
& \leq C\left(\left|p^{0, H}\right|_{H^{1}(\Omega)}+\left\|\mathbf{f}^{H}\right\|_{L^{2}(\Omega)}\right) \sup _{x \in Q^{H}}\left\|\bar{a}(x)-a^{0}(x)\right\|_{\mathrm{F}} \\
& \leq C\left\|\mathbf{f}^{H}\right\|_{L^{2}(\Omega)} \sup _{x \in Q^{H}}\left\|\bar{a}(x)-a^{0}(x)\right\|_{\mathrm{F}},
\end{aligned}
$$

where $C$ depends only on the constants $\lambda$ and $\Lambda$ from the assumptions. Further, one has

$$
\left\|\mathbf{u}^{0, H}-\overline{\mathbf{u}}^{H}\right\|_{L^{2}(\Omega)} \leq C\left|p^{0, H}-\bar{p}^{H}\right|_{H^{1}(\Omega)} .
$$

If we use DS-FE-HMM with periodic micro boundary conditions and explicit scale separation $\left(\delta=\varepsilon\right.$ and $\left.Y_{\mathrm{F}}^{x, \delta}=Y_{\mathrm{F}}^{x}\right)$, then this error vanishes.

4.4. Micro error. This error arises from the FE approximation of the Stokes problem on the micro scale. We can use the same strategy as for the modeling error to show

$$
\begin{aligned}
\left|\bar{p}^{H}-p^{H}\right|_{H^{1}(\Omega)} & \leq C\left\|\mathbf{f}^{H}\right\|_{L^{2}(\Omega)} \sup _{x \in Q^{H}}\left\|\bar{a}(x)-a^{h}(x)\right\|_{\mathrm{F}}, \\
\left\|\overline{\mathbf{u}}^{H}-\mathbf{u}^{H}\right\|_{H^{1}(\Omega)} & \leq C\left|\bar{p}^{H}-p^{H}\right|_{H^{1}(\Omega)},
\end{aligned}
$$

where $C$ depends only on the ellipticity and boundedness constants of the bilinear form $B_{H}(\cdot, \cdot)$. Using the definitions of $\bar{a}$ and $a^{h}$ and the Cauchy-Schwarz inequality, we obtain

$$
\begin{aligned}
\left\|\bar{a}(x)-a^{h}(x)\right\|_{\mathrm{F}}^{2} & =\left(\sum_{i, j=1}^{d} \frac{\varepsilon^{d}}{\delta^{d}} \int_{Y_{\mathrm{F}}^{x, \delta}}\left(\overline{\mathbf{u}}_{j}^{i, x}-\mathbf{u}_{j}^{i, x, h}\right) \mathrm{d} y\right)^{2} \\
& \leq \sum_{i=1}^{d} \frac{\varepsilon^{d}}{\delta^{d}}\left\|\overline{\mathbf{u}}^{i, x}-\mathbf{u}^{i, x, h}\right\|_{L^{2}\left(Y_{\mathrm{F}}^{x, \delta}\right)}^{2}
\end{aligned}
$$

Hence, the micro error can be bounded using the $L^{2}$-norm of the error of the micro problems. Using a priori convergence estimates for Stokes problem (see $[18,33,52]$ ), one can obtain

$$
\left|\bar{p}^{H}-p^{H}\right|_{H^{1}(\Omega)} \leq C h^{\theta} \cdot
$$

where $C$ does not depend on the mesh sizes $H$ and $h$. The constant $\theta$ depends on the regularity of the micro problem. Optimally, one has $\theta=k+2$ for the $\mathbb{P}_{k+1} / \mathbb{P}_{k}$ Taylor-Hood FEs or $\theta=2$ for MINI FEs (see [18]). 
5. A posteriori error estimates. There are tow major issues for the DS-FE-HMM method (25) when using uniform mesh refinement on the macro and the micro scales. First, it is well-known that for non-convex macro domain $\Omega$ the optimal convergence rates (47) will deteriorate. Second, the DS-FE-HMM accuracy will also deteriorate if the approximation of the effective tensor $a^{h}(x)$ is not accurate enough, leading to a large micro error. However, the domains $Y_{\mathrm{F}}^{x, \delta}$ are usually not convex for porous medium so the regularity of the micro problems is low. For example, in two dimensions the exponent from $(49)$ satisfies $\theta \in(1,2)$ depending on the maximal interior angle of $Y_{\mathrm{F}}^{x, \delta}$ (see [52]).

We can thus not rely on a priori error analysis in general to develop a robust approximation of flow in porous medium. We therefore propose an adaptive method for both the macro and the micro solvers. To drive the coupled adaptive mesh refinement we need therefore to derive rigorous a posteriori error estimates on both scales and derive an algorithm to adequately balance the macro and micro mesh refinement. Inspired by $[4,6]$ we prove reliability and efficiency of the multiscale macro residual. We then define the multiscale micro residual and prove the reliability of the combined multiscale macro-micro residuals.

5.1. Notations, auxiliary identities and inequalities. Denote the set of all edges of triangles of $\mathcal{T}_{H}$ by $\mathcal{E}_{H}$. For any edge $e \in \mathcal{E}_{H}$ or element $K \in \mathcal{T}_{H}$ set

$$
\begin{aligned}
N(K) & =\left\{T \in \mathcal{T}_{H} ; \bar{K} \cap \bar{T} \neq \emptyset\right\}, \quad M(K)=\left\{T \in \mathcal{T}_{H} ; K=T \text { or } \partial K \cap \partial T \in \mathcal{E}_{H}\right\}, \\
N(e) & =\left\{T \in \mathcal{T}_{H} ; \bar{e} \cap \bar{T} \neq \emptyset\right\} .
\end{aligned}
$$

If $e \in \mathcal{E}_{H}$ is a common edge of two distinct elements $K, T \in \mathcal{T}_{H}$, then [- $]_{e}$ denotes the jump of a (possibly discontinuous) quantity over the edge $e$. If $\mathbf{v}$ is a vector field with $\left.\mathbf{v}\right|_{K},\left.\mathbf{v}\right|_{T} \in C^{0}$, then

$$
[\mathbf{v} \cdot \mathbf{n}]_{e}(x)=\left.\mathbf{v}\right|_{K}(x) \cdot \mathbf{n}_{K}(x)+\left.\mathbf{v}\right|_{T}(x) \cdot \mathbf{n}_{T}(x) .
$$

If $e \subset \partial K \cap \partial \Omega$, we assume an artificial element $T$ on the other side of $e$ and define $\left.\mathbf{v}\right|_{T} \equiv 0$.

We will denote by $I^{H}: H^{1}(\Omega) \rightarrow S_{H}^{1}(\Omega)$ the Clément interpolation operator [25] and recall the inverse inequality (see [24, Thm. 3.2.6])

$$
\left|q^{H}\right|_{H^{1}(K)} \leq C H_{K}^{-1}\left\|q^{H}\right\|_{L^{2}(K)}
$$

for any $q^{H} \in S_{H}^{l}(\Omega)$ and $K \in \mathcal{T}_{H}$, where $C$ depends only on $d, l$, and the shape-regularity of $K$. For any $q \in H^{1}(\Omega), K \in \mathcal{T}_{H}$, and $e \in \mathcal{E}_{H}$, we have (see [8, Thm. 3.10]) the trace inequality

$$
\|q\|_{L^{2}(e)} \leq C H_{e}^{1 / 2}|q|_{H^{1}(K)}+C H_{e}^{-1 / 2}\|q\|_{L^{2}(K)}
$$

where $H_{e}=\operatorname{diam}(e)$ and the interpolation estimates (see [25])

$$
\begin{aligned}
\left\|q-I^{H} q\right\|_{L^{2}(K)} & \leq C H_{K}|q|_{H^{1}(N(K))}, \\
\left|q-I^{H} q\right|_{H^{1}(K)} & \leq C|q|_{H^{1}(N(K))}, \\
\left\|q-I^{H} q\right\|_{L^{2}(e)} & \leq C H_{e}^{1 / 2}|q|_{H^{1}(N(e))},
\end{aligned}
$$

where $C$ depends only on $d$ and the shape-regularity of $\mathcal{T}_{H}$.

5.2. Residual-based error estimates. Our goal is to find an a posteriori error estimate of $e=p^{0}-p^{H}$ in the $H_{1}$-seminorm and prove efficiency of these bounds. Let

$$
\begin{aligned}
& \xi_{K}^{2}=\left\|a^{0}\left(\mathbf{f}-\nabla p^{H}\right)-\Pi_{a^{h}}\left(\mathbf{f}^{H}-\nabla p^{H}\right)\right\|_{L^{2}(K)}^{2}, \\
& \eta_{K}^{2}=H_{K}^{2}\left\|\nabla \cdot \Pi_{a^{h}}\left(\mathbf{f}^{H}-\nabla p^{H}\right)\right\|_{L^{2}(K)}^{2}+\sum_{e \in \partial K} \frac{1}{2} H_{e}\left\|\left[\Pi_{a^{h}}\left(\mathbf{f}^{H}-\nabla p^{H}\right) \cdot \mathbf{n}\right]_{e}\right\|_{L^{2}(e)}^{2}
\end{aligned}
$$

for any $K \in \mathcal{T}_{H}$. If $K^{\prime}$ is any union of elements $K$ in $\mathcal{T}_{H}$, e.g., $K^{\prime}=\Omega$, we define $\xi_{K^{\prime}}^{2}=$ $\sum_{K \subset K^{\prime}} \xi_{K}^{2}$ and $\eta_{K^{\prime}}^{2}=\sum_{K \subset K^{\prime}} \eta_{K}^{2}$.

Theorem 27. Assume that $a^{0}(x) \xi \cdot \xi \geq \lambda|\xi|^{2}$ and $\left|a^{0}(x) \xi\right| \leq \Lambda|\xi|$ for each $\xi \in \mathbb{R}^{d}$ and a.e. $x \in \Omega$. Then there exists a constant $C$ depending only on $\Omega, \lambda$, and the shape-regularity of $\mathcal{T}_{H}$ such that

$$
\left|p^{H}-p^{0}\right|_{H^{1}(\Omega)}^{2} \leq C\left(\eta_{\Omega}^{2}+\xi_{\Omega}^{2}\right)
$$

and a constant $C$ depending only on $\Omega, d, l, \Lambda$, and the shape-regularity of $\mathcal{T}_{H}$ such that for any $K \in \mathcal{T}_{H}$ we have

$$
\eta_{K}^{2} \leq C\left(\left|p^{H}-p^{0}\right|_{H^{1}(M(K))}^{2}+\xi_{M(K)}^{2}\right)
$$

The proof of Theorem 27 follows closely [7,37] and is divided into two parts. 
Part 1: Upper bound. Let us state and prove an error representation formula.

Lemma 28. For any $q \in H^{1}(\Omega) / \mathbb{R}$ and any $q^{H} \in S_{H}^{l}(\Omega) / \mathbb{R}$ we have

$$
\begin{aligned}
B_{0}(e, q) & =\sum_{K \in \mathcal{T}_{H}} \int_{K}\left(a^{0}\left(\mathbf{f}-\nabla p^{H}\right)-\Pi_{a^{h}}\left(\mathbf{f}^{H}-\nabla p^{H}\right)\right) \cdot \nabla q \mathrm{~d} x \\
& +\sum_{e \in \mathcal{E}_{H}} \int_{e}\left[\Pi_{a^{h}}\left(\mathbf{f}^{H}-\nabla p^{H}\right) \cdot \mathbf{n}\right]_{e}\left(q-q^{H}\right) \mathrm{d} s \\
& -\sum_{K \in \mathcal{T}_{H}} \int_{K}\left(\nabla \cdot \Pi_{a^{h}}\left(\mathbf{f}^{H}-\nabla p^{H}\right)\right)\left(q-q^{H}\right) \mathrm{d} x .
\end{aligned}
$$

Proof. Using (10) and (11) for any $q \in H^{1}(\Omega) / \mathbb{R}$ we get

$$
\begin{aligned}
B_{0}(e, q) & =\sum_{K \in \mathcal{T}_{H}} \int_{K}\left(a^{0}\left(\mathbf{f}-\nabla p^{H}\right)-\Pi_{a^{h}}\left(\mathbf{f}^{H}-\nabla p^{H}\right)\right) \cdot \nabla q \mathrm{~d} x \\
& +\sum_{K \in \mathcal{T}_{H}} \int_{K} \Pi_{a^{h}}\left(\mathbf{f}^{H}-\nabla p^{H}\right) \cdot \nabla q \mathrm{~d} x .
\end{aligned}
$$

The integration by parts formula

$$
\begin{aligned}
\sum_{K \in \mathcal{T}_{H}} \int_{K} \Pi_{a^{h}}\left(\mathbf{f}^{H}-\nabla p^{H}\right) \cdot \nabla q \mathrm{~d} x= & \sum_{e \in \mathcal{E}_{H}} \int_{e}\left[\Pi_{a^{h}}\left(\mathbf{f}^{H}-\nabla p^{H}\right) \cdot \mathbf{n}\right]_{e} q \mathrm{~d} s \\
& -\sum_{K \in \mathcal{T}_{H}} \int_{K}\left(\nabla \cdot \Pi_{a^{h}}\left(\mathbf{f}^{H}-\nabla p^{H}\right)\right) q \mathrm{~d} x
\end{aligned}
$$

yields

$$
\begin{aligned}
B_{0}(e, q) & =\sum_{K \in \mathcal{T}_{H}} \int_{K}\left(a^{0}\left(\mathbf{f}-\nabla p^{H}\right)-\Pi_{a^{h}}\left(\mathbf{f}^{H}-\nabla p^{H}\right)\right) \cdot \nabla q \mathrm{~d} x \\
& +\sum_{e \in \mathcal{E}_{H}} \int_{e}\left[\Pi_{a^{h}}\left(\mathbf{f}^{H}-\nabla p^{H}\right) \cdot \mathbf{n}\right]_{e} q \mathrm{~d} s-\sum_{K \in \mathcal{T}_{H}} \int_{K}\left(\nabla \cdot \Pi_{a^{h}}\left(\mathbf{f}^{H}-\nabla p^{H}\right)\right) q \mathrm{~d} x .
\end{aligned}
$$

Using $L_{H}\left(q^{H}\right)-B_{H}\left(p^{H}, q^{H}\right)=0$ for any $q^{H} \in S_{H}^{l}(\Omega) / \mathbb{R}$, formulas (29), (30), and integration by parts, gives

$$
0=\sum_{e \in \mathcal{E}_{H}} \int_{e}\left[\Pi_{a^{h}}\left(\mathbf{f}^{H}-\nabla p^{H}\right) \cdot \mathbf{n}\right]_{e} q^{H} \mathrm{~d} s-\sum_{K \in \mathcal{T}_{H}} \int_{K}\left(\nabla \cdot \Pi_{a^{h}}\left(\mathbf{f}^{H}-\nabla p^{H}\right)\right) q^{H} \mathrm{~d} x
$$

Subtracting (56) from (55) we get the desired result.

Let $q=e$ and $q^{H}=I^{H} e$ in Lemma 28 and use the Cauchy-Schwarz inequality to get

$$
\begin{aligned}
B_{0}(e, e) \leq & \sum_{K \in \mathcal{T}_{H}}\left\|a^{0}\left(\mathbf{f}-\nabla p^{H}\right)-\Pi_{a^{h}}\left(\mathbf{f}^{H}-\nabla p^{H}\right)\right\|_{L^{2}(K)}|e|_{H^{1}(K)} \\
& +\sum_{e \in \mathcal{E}_{H}}\left\|\left[\Pi_{a^{h}}\left(\mathbf{f}^{H}-\nabla p^{H}\right) \cdot \mathbf{n}\right]_{e}\right\|_{L^{2}(e)}\left\|e-I^{H} e\right\|_{L^{2}(e)} \\
& +\sum_{K \in \mathcal{T}_{H}}\left\|\nabla \cdot \Pi_{a^{h}}\left(\mathbf{f}^{H}-\nabla p^{H}\right)\right\|_{L^{2}(K)}\left\|e-I^{H} e\right\|_{L^{2}(K)} .
\end{aligned}
$$

Using the interpolation results (51) and the Cauchy-Schwarz inequality then yields

$$
B_{0}(e, e) \leq C\left(\xi_{\Omega}^{2}+\eta_{\Omega}^{2}\right)^{1 / 2}|e|_{H^{1}(\Omega)},
$$

where $C$ depends only on $d$ and the shape-regularity of $\mathcal{T}_{H}$, using the finite overlapping property of the neighborhoods $N(K)$. Combining (57) and the uniform ellipticity of $a^{0}(x)$ proves (54). 
Part 2: Lower Bound. We derive two estimates related to the interior and to the jump parts of the residual $\eta_{K}$. The result (54) then follows by combining the inequalities (58) and (63) for all $e \in \partial K$.

Interior Residual. Let $K \in \mathcal{T}_{H}$ and $\Psi_{K}$ be the standard bubble function for element $K$, i.e., $\Psi_{K} \in S_{H}^{d+1}(\Omega)$ such that $\left.\Psi_{K}\right|_{\Omega \backslash K} \equiv 0$ and $\Psi_{K}\left(x_{K \text {,bary }}\right)=1$ at the barycenter $x_{K \text {,bary }}$ of $K$. We next use the representation formula of Lemma 28 with $q=\Psi_{K} \nabla \cdot \Pi_{a^{h}}\left(\mathbf{f}^{H}-\nabla p^{H}\right)$ and $q^{H}=0$ to obtain

$\int_{K} \Psi_{K}\left(\nabla \cdot \Pi_{a^{h}}\left(\mathbf{f}^{H}-\nabla p^{H}\right)\right)^{2} \mathrm{~d} x=-B_{0}(e, q)+\int_{K}\left(a^{0}\left(\mathbf{f}-\nabla p^{H}\right)-\Pi_{a^{h}}\left(\mathbf{f}^{H}-\nabla p^{H}\right)\right) \cdot \nabla q \mathrm{~d} x$.

Using the continuity of $a^{0}$, the Cauchy-Schwarz inequality, and the equivalence of norms $\|\nu\|_{L^{2}(K)}$ and $\left(\int_{K} \Psi_{K} \nu^{2} \mathrm{~d} x\right)^{1 / 2}$ for $\nu \in \mathcal{P}^{l-1}(K)$ (see e.g., [9, Theorem 3.3]), we obtain

$$
\left\|\nabla \cdot \Pi_{a^{h}}\left(\mathbf{f}^{H}-\nabla p^{H}\right)\right\|_{L^{2}(K)}^{2} \leq C\left(|e|_{H^{1}(K)}+\xi_{K}\right)|q|_{H^{1}(K)},
$$

where we have used the definition (52) for $\xi_{K}$. Using the inverse inequality (50) for $q$ and the property $\left|\Psi_{K}\right| \leq 1$ leads to

$$
H_{K}^{2}\left\|\nabla \cdot \Pi_{a^{h}}\left(\mathbf{f}^{H}-\nabla p^{H}\right)\right\|_{L^{2}(K)}^{2} \leq C\left(|e|_{H^{1}(K)}^{2}+\xi_{K}^{2}\right) .
$$

Jump Residual. We set $q^{H} \equiv 0$ in Lemma 28, then use the Cauchy-Schwarz inequality and continuity of $B_{0}$ to obtain

$$
\begin{array}{r}
\sum_{e \in \mathcal{E}_{H}} \int_{e}\left[\Pi_{a^{h}}\left(\mathbf{f}^{H}-\nabla p^{H}\right) \cdot \mathbf{n}\right]_{e} q \mathrm{~d} s \leq C \sum_{K \in \mathcal{T}_{H}}\left(\left\|\nabla \cdot \Pi_{a^{h}}\left(\mathbf{f}^{H}-\nabla p^{H}\right)\right\|_{L^{2}(K)}\|q\|_{L^{2}(K)}\right. \\
\left.+|e|_{H^{1}(K)}|q|_{H^{1}(K)}+\xi_{K}|q|_{H^{1}(K)}\right) .
\end{array}
$$

Let $K \in \mathcal{T}_{H}, e \in \partial K$, and $\Psi_{e} \in \mathcal{P}^{d}(e)$ be the bubble function on $e$, i.e., $\left.\Psi_{e}\right|_{\partial e} \equiv 0$ and $\Psi_{e}\left(e_{\text {bary }}\right)=1$ at the barycenter $e_{\text {bary }}$ of $e$. Define function $q^{e}=\Psi_{e}\left[\Pi_{a^{h}}\left(\mathbf{f}^{H}-\nabla p^{H}\right) \cdot \mathbf{n}\right]_{e}$ and notice that $q^{e} \in \mathcal{P}_{0}^{d+l-1}(e)=\left\{r \in \mathcal{P}^{d+l-1}(e) ;\left.r\right|_{\partial e} \equiv 0\right\}$.

By [16, Chap. XI, Lemma 2.7], there is a lifting operator $\mathcal{R}_{K, e}: \mathcal{P}_{0}^{d+l-1}(e) \rightarrow \mathcal{P}^{d+l-1}(K)$ such that $\left.\mathcal{R}_{K, e}\left(q^{e}\right)\right|_{e}=\left.q^{e}\right|_{e}$ and $\left.\mathcal{R}_{K, e}\left(q^{e}\right)\right|_{\partial K \backslash e}=0$. Moreover, we have

$$
\left|\mathcal{R}_{K, e}\left(q^{e}\right)\right|_{H^{1}(K)}+H_{K}^{-1}\left\|\mathcal{R}_{K, e}\left(q^{e}\right)\right\|_{L^{2}(K)} \leq C H_{e}^{-1 / 2}\left\|q^{e}\right\|_{L^{2}(e)},
$$

where $C$ depends only on $d, l$, and the shape-regularity of $\mathcal{T}_{H}$. For any interior interface $e \in \mathcal{E}_{H}$, let $K_{1}$ and $K_{2}$ be two elements such that $e=\partial K_{1} \cap \partial K_{2}$ and define

$$
q= \begin{cases}\mathcal{R}_{K_{i}, e}\left(q^{e}\right) & \text { in } K_{i} \text { for } i=1,2, \\ 0 & \text { elsewhere in } \Omega .\end{cases}
$$

Using this function $q$ in (59) together with the inequality (60) gives

$$
\begin{aligned}
\int_{e} \Psi_{e}\left[\Pi_{a^{h}}\left(\mathbf{f}^{H}-\nabla p^{H}\right) \cdot \mathbf{n}\right]_{e}^{2} \mathrm{~d} s \leq & C H_{e}^{-1 / 2}\left\|q^{e}\right\|_{L^{2}(e)} \sum_{i=1,2}\left(\xi_{K_{i}}+|e|_{H^{1}\left(K_{i}\right)}\right. \\
& \left.+H_{K_{i}}\left\|\nabla \cdot \Pi_{a^{h}}\left(\mathbf{f}^{H}-\nabla p^{H}\right)\right\|_{L^{2}\left(K_{i}\right)}\right) .
\end{aligned}
$$

Using the the property $\left|\Psi_{e}\right| \leq 1$ and the equivalence of norms $\|\nu\|_{L^{2}(e)}$ and $\left(\int_{e} \Psi_{e} \nu^{2} \mathrm{~d} s\right)^{1 / 2}$ in (61) yields

$$
\begin{aligned}
H_{e}\left\|\left[\Pi_{a^{h}}\left(\mathbf{f}^{H}-\nabla p^{H}\right) \cdot \mathbf{n}\right]_{e}\right\|_{L^{2}(e)}^{2} \leq C & \sum_{m=1,2}\left(\xi_{K_{m}}^{2}+|e|_{H^{1}\left(K_{m}\right)}^{2}\right. \\
& \left.+H_{K_{m}}^{2}\left\|\nabla \cdot \Pi_{a^{h}}\left(\mathbf{f}^{H}-\nabla p^{H}\right)\right\|_{L^{2}\left(K_{m}\right)}^{2}\right) .
\end{aligned}
$$

The last step is to use (58) in (62) and obtain

$$
H_{e}\left\|\left[\Pi_{a^{h}}\left(\mathbf{f}^{H}-\nabla p^{H}\right) \cdot \mathbf{n}\right]_{e}\right\|_{L^{2}(e)}^{2} \leq C \sum_{m=1,2}\left(\xi_{K_{m}}^{2}+|e|_{1, K_{m}}^{2}\right) .
$$

Finally, combining the estimate for the interior residual (58) and the jump residual (63) gives the lower bound (54). 
5.3. A posteriori analysis of the micro error. Let $K \in \mathcal{T}_{H}$ be arbitrary and use the triangle inequality to obtain the decomposition $\xi_{K} \leq \xi_{\text {data }, K}+\xi_{\text {mic }, K}$, where

$$
\xi_{\text {data }, K}^{2}=\left\|a^{0}\left(\mathbf{f}-\nabla p^{H}\right)-\Pi_{\bar{a}}\left(\mathbf{f}^{H}-\nabla p^{H}\right)\right\|_{L^{2}(K)}^{2}, \quad \xi_{\text {mic }, K}^{2}=\left\|\Pi_{a^{h}-\bar{a}}\left(\mathbf{f}^{H}-\nabla p^{H}\right)\right\|_{L^{2}(K)}^{2} .
$$

Recall the definition of $Q^{K}$ in (21). A simple estimation gives

$$
\begin{aligned}
\xi_{\text {mic }, K}^{2} & =\sum_{j=1}^{J} \omega_{K_{j}}\left|\left(a^{h}\left(x_{K_{j}}\right)-\bar{a}\left(x_{K_{j}}\right)\right)\left(\mathbf{f}^{H}\left(x_{K_{j}}\right)-\nabla p^{H}\left(x_{K_{j}}\right)\right)\right|^{2} \\
& \leq\left\|\mathbf{f}^{H}-\nabla p^{H}\right\|_{L^{2}(K)}^{2} \max _{x \in Q^{K}}\left\|a^{h}(x)-\bar{a}(x)\right\|_{\mathrm{F}}^{2} .
\end{aligned}
$$

The inequality (64) is a foundation for the a posteriori estimation of the micro error $\xi_{\text {mic }, K}$. Using (48) and the Poincaré inequality gives

$$
\xi_{\text {mic }, K}^{2} \leq C\left(\frac{\varepsilon}{\delta}\right)^{d}\left\|\mathbf{f}^{H}-\nabla p^{H}\right\|_{L^{2}(K)}^{2} \max _{x \in Q^{K}} \sum_{i=1}^{d}\left|\mathbf{u}^{i, x, h}-\overline{\mathbf{u}}^{i, x}\right|_{H^{1}\left(Y_{\mathrm{F}}^{x, \delta}\right)}^{2},
$$

where $C$ depends only on the bound $\alpha$ for the continuity of $a^{h}$ (see Section 3.2). The micro FE error $\left|\mathbf{u}^{i, x, h}-\overline{\mathbf{u}}^{i, x}\right|_{H^{1}\left(Y_{\mathrm{F}}^{x, \delta}\right)}$ can be estimated using the classical residual-based error estimator for Stokes problem, see [53, Theorem 3.1]. For any quadrature point $x \in Q^{K}$ there exists a constant $C$ that depends only on the inf-sup and Poincaré-Friedrichs constants of the Stokes micro problem, shape regularity of $\mathcal{T}_{h}^{x}$, and the micro FE type such that for any $i \in\{1, \ldots, d\}$ we have

$$
C^{-1} \eta_{\text {stokes }, x, i}^{2} \leq\left|\mathbf{u}^{i, x, h}-\overline{\mathbf{u}}^{i, x}\right|_{H^{1}\left(Y_{\mathrm{F}}^{x, \delta}\right)}^{2}+\left\|p^{i, x, h}-\bar{p}^{i, x}\right\|_{L^{2}\left(Y_{\mathrm{F}}^{x, \delta}\right) / \mathbb{R}}^{2} \leq C \eta_{\text {stokes }, x, i}^{2}
$$

where

$$
\begin{aligned}
\eta_{\text {stokes }, x, i}^{2}= & \sum_{T \in \mathcal{T}_{h}^{x}}\left(\sum_{e \in \partial T \cap \mathcal{E}_{h}^{x}} \frac{H_{e}}{2}\left\|\left[\frac{\partial \mathbf{u}^{i, x, h}}{\partial \mathbf{n}}-p^{i, x, h} \mathbf{n}\right]_{e}\right\|_{L^{2}(e)}^{2}\right. \\
& \left.+h_{T}^{2}\left\|\Delta \mathbf{u}^{i, x, h}-\nabla p^{i, x, h}+\mathbf{e}^{i}\right\|_{L^{2}(T)}^{2}+\left\|\nabla \cdot \mathbf{u}^{i, x, h}\right\|_{L^{2}(T)}^{2}\right)
\end{aligned}
$$

and $\mathcal{E}_{h}^{x}$ is the set of all edges in $\mathcal{T}_{h}^{x}$ except the Dirichlet boundary edges. Notice that (66) does not contain any data approximation error, since the force term in (27) is constant. Applying (66) in (65) yields $\xi_{\text {mic }, K}^{2} \leq C \eta_{\text {mic }, K}^{2}$, where

$$
\eta_{\text {mic }, K}^{2}=(\varepsilon / \delta)^{d}\left\|\mathbf{f}^{H}-\nabla p^{H}\right\|_{L^{2}(K)}^{2} \max _{x \in Q^{K}} \sum_{i=1}^{d} \eta_{\text {stokes }, x, i}^{2}
$$

As before, we set $\eta_{\text {mic }, \Omega}^{2}=\sum_{K \in \mathcal{T}_{H}} \eta_{\text {mic }, K}^{2}$ and $\xi_{\text {data }, \Omega}^{2}=\sum_{K \in \mathcal{T}_{H}} \xi_{\text {data }, K}^{2}$.

Theorem 29. There is a constant $C$ depending only on the domain $\Omega$, the continuity $(\Lambda)$ and coercivity $(\lambda)$ constants of $a^{0}(x)$, the degree $l$ of the macro finite element, the shape-regularity of $\mathcal{T}_{H}$ and the constant $C$ in (66) such that

$$
\left|p^{0}-p^{H}\right|_{H^{1}(\Omega)}^{2} \leq C\left(\eta_{\Omega}^{2}+\eta_{m i c, \Omega}^{2}+\xi_{\text {data }, \Omega}^{2}\right) .
$$

6. Adaptive algorithm. We propose an adaptive numerical algorithm for the DS-FEHMM problem (25), (27), (28). The individual macro and micro adaptive processes follow the standard FEM refinement cycle

$$
\longrightarrow \mathrm{SOLVE} \rightarrow \text { ESTIMATE } \rightarrow \text { MARK } \rightarrow \text { REFINE. } \square
$$

Our main goal is to optimally couple the macro and micro mesh refinements. To accomplish that, we enforce

$$
\eta_{\text {mic }, K}^{2} \leq \mu \eta_{K}^{2} \quad \forall K \in \mathcal{T}_{H},
$$

where the constant $\mu>0$ is problem dependent and can be calibrated as described in Remark 30. While solving a micro problem (27), we refine the micro mesh $\mathcal{T}_{h}^{x}$ until the condition

$$
\eta_{\text {stokes }, x, i}^{2} \leq \frac{\mu}{d} \eta_{K}^{2}\left\|\mathbf{f}^{H}-\nabla p^{H}\right\|_{L^{2}(K)}^{-2},
$$

is met, which implies (69). 


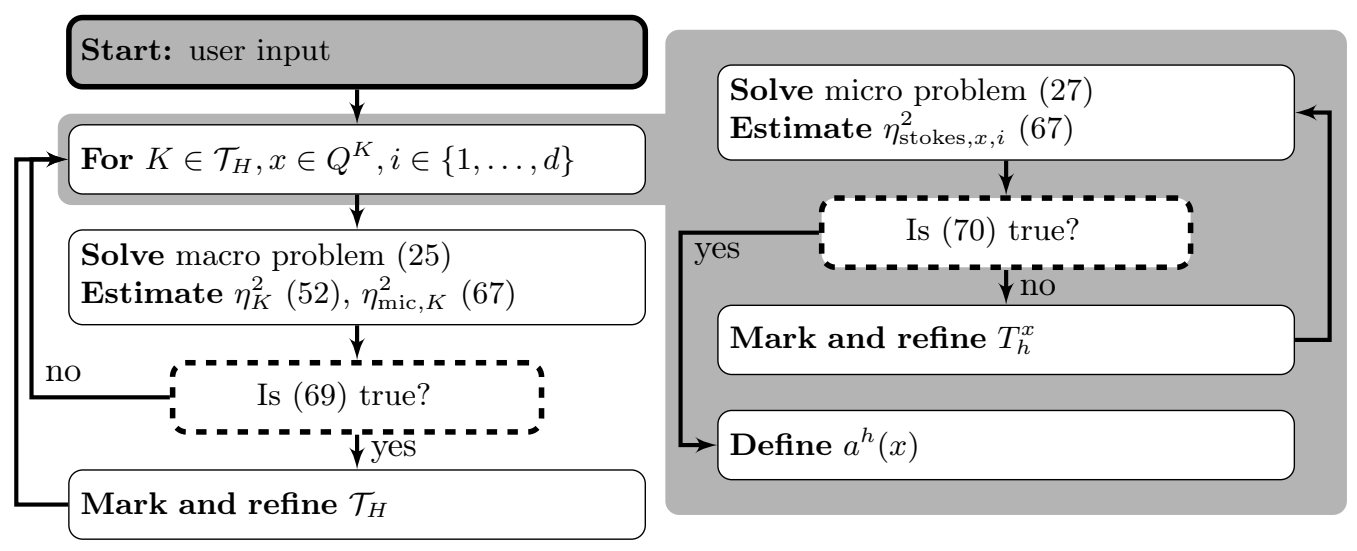

Figure 9: A flow-chart of the adaptive DS-FE-HMM algorithm for the Stokes problem.

Algorithm. We assume that the user provides $\Omega, \Omega_{\varepsilon}, \delta$, an initial macro mesh $\mathcal{T}_{H}$ of $\Omega$, finite element spaces, and the micro coupling (periodic or Neumann). The adaptive procedure that is depicted as a flow chart in Figure 6 consists in the following steps:

Solve. Solve for each quadrature point $x \in Q^{H}$ the Stokes micro problems (27) adaptively using the stopping criterion (70) (using $p^{H}$ and $\eta_{K}$ from the previous step). Assemble and solve the macro elliptic problem (25).

Estimate Compute $\eta_{K}$ and $\eta_{\text {mic }, K}$ and repeat the previous step until (69) is satisfied.

Mark. Mark a subset of the elements in $\mathcal{T}_{H}$ by using the indicator $\eta_{K}$ (we use the marking strategy E [54, Chapter 4.1]).

Refine. The marked elements are refined so as to guarantee the conformity and shape-regularity of the refined meshes.

An efficient implementation of the proposed algorithm must contain a mechanism for saving and reusing the data from the micro problems. We propose to store for each quadrature point $x \in Q^{H}$ the values $a^{h}(x), \eta_{\text {stokes }, x, i}^{2}$, the most refined micro mesh $\mathcal{T}_{h}^{x}$ that was reached and the corresponding micro solution. Since we can verify (69) only after all the micro problems (and the macro problem) are computed, it occurs that one needs to solve some micro problems with higher precision. If the finest solution and the finest mesh of a micro problem is saved, then they can be reused as a starting point for the additional refinement cycles.

Remark 30. The exact value of $\mu$ is not crucial for the efficiency of the algorithm, only its order of magnitude matters. In an offline stage we estimate $e_{\mathrm{mic}}$ and $e_{\mathrm{mac}}$ by performing a few iterations of uniform refinements in the DS-FE-HMM (at the macro and the micro-level) and denote $\tilde{e}_{\text {mic }}$ and $\tilde{e}_{\text {mac }}$ these approximations and $\tilde{\eta}_{\Omega}, \tilde{\eta}_{\text {mic }, \Omega}$ the corresponding residuals. We then set

$$
\mu=\frac{\left|\tilde{e}_{\mathrm{mac}}\right|_{H^{1}(\Omega)}^{2}}{\tilde{\eta}_{\Omega}^{2}} \cdot \frac{\eta_{\mathrm{mic}, \Omega}^{2}}{\left|\tilde{e}_{\mathrm{mic}}\right|_{H^{1}(\Omega)}^{2}} .
$$

We note that the value of $\mu$ can be updated during the adaptive DS-FE-HMM.

The marking strategy E contains one parameter that is usually denoted $\theta$. We use $\theta=0.5$ for the micro problems and $\theta=0.25$ for the macro problem. To guarantee conformity and shape-regularity of the refined meshes, we use the newest vertex bisection in two dimensions and the modified longest edge bisection [14] in three dimensions.

7. Numerical Experiments. In this section, we present numerical experiments that test the capabilities of the adaptive DS-FE-HMM. Three different non-periodic porous media, called $A, B$ and $C$, are presented. They are all based on a locally periodic porous geometry as described in Section 2.3. The two-dimensional medium $A$ has a simple pore geometry and is used to demonstrate the convergence rates of various element types. We also test the multiscale method for different boundary conditions and sizes of the representative domains in 
the Stokes micro problems. The two-dimensional medium $B$ illustrates the performance of the method on a more complex porous material. We conclude this section by a three-dimensional experiment performed on the medium $C$.

All the numerical computations were performed in Matlab with FE code inspired by [5] and the AFEM code [23] with mesh generation provided by gmsh [32]. Linear systems were solved using Matlab's mldivide for $d=2$. In three dimensions, we used algebraic multigrid solver AGMG [38] for positive definite (macro) problems and an Uzawa method [41] for saddle point (micro) problems. The Uzawa method uses algebraic multigrid preconditioning for the coercive part and pressure mass matrix preconditioning for the Shur's complement.

7.1. Porous medium $A$. Consider the macro domain $\Omega=((0,2) \times(0,3)) \backslash([1,2] \times[1,2])$ with periodic boundary conditions that connect the edges $(0,2) \times\{0\}$ and $(0,2) \times\{3\}$ and the force field $\mathbf{f} \equiv \mathbf{f}^{H} \equiv(0,-1)$ as shown in Figure 10 .

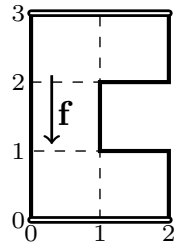

$\Omega$

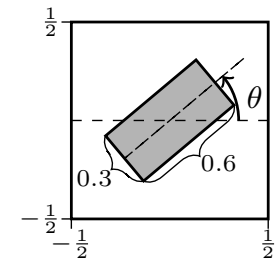

medium $A$

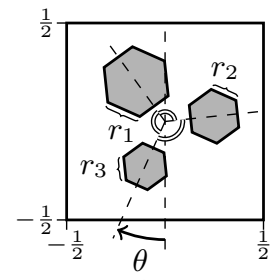

medium $B$

Figure 10: $\Omega$ and pore geometries $\left(Y_{\mathrm{S}}^{x}, Y_{\mathrm{F}}^{x}\right)$ for the media $A$ and $B$ (gray solid part).

We define $Y_{\mathrm{S}}^{x}$ to be a closed rectangle of size $0.6 \times 0.3$ centered in the middle of $Y$ and rotated by the angle $\theta(x)=\left(1-x_{1}^{2} / 8-x_{2} / 3\right) \pi$. The mapping $\varphi$ can be appropriately defined to satisfy $\varphi\left(x, Y_{\mathrm{S}}\right)=Y_{\mathrm{S}}^{x}$, where $Y_{\mathrm{S}}$ is $Y_{\mathrm{S}}^{x}$ for $x=[0,0]$. A sketch of the pore geometry is given in Figure 10 and examples of the micro problem solutions are plotted in Figure 11.
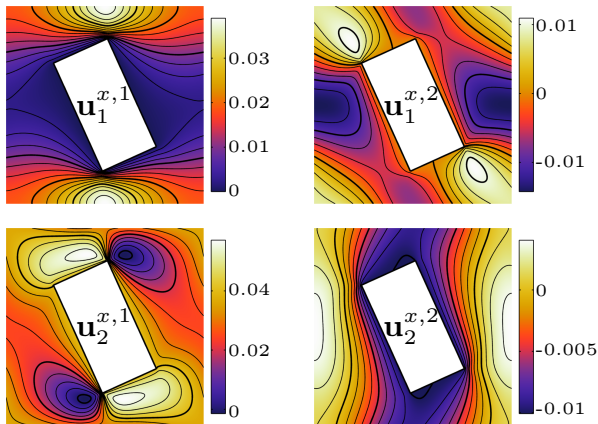

$$
a^{0}(x) \approx 10^{-3}\left(\begin{array}{cc}
9.761 & -1.898 \\
-1.898 & 24.085
\end{array}\right)
$$
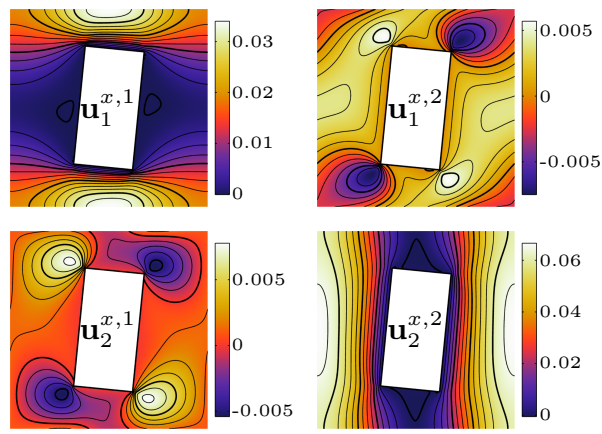

$$
a^{0}(x) \approx 10^{-3}\left(\begin{array}{cc}
9.036 & 0.685 \\
0.685 & 31.026
\end{array}\right)
$$

Figure 11: Medium $A$ : micro velocity solutions for $x=[0.5,1]$ (left) and $x=[0.5,1.5]$ (right).

Porous domains $\Omega_{\varepsilon}$ and fine scale solution $p^{\varepsilon}$ are plotted in Figure 12 for various values of $\varepsilon$. The solutions $p^{\varepsilon}$ were computed numerically using single-scale adaptive FEM with $\mathbb{P}_{2} / \mathbb{P}_{1}$ FEs. This is a costly computation and we therefore limited such a fine scale solve to $\varepsilon \geq 2^{-4}$.

For the numerical multiscale method we set $\varepsilon=\delta=10^{-4}$ and use periodic BC on micro problems. We take $\mathbb{P}_{1}$ macro FEs $(l=1)$ and $\mathbb{P}_{2} / \mathbb{P}_{1}$ micro FEs $(k=1)$. The initial macro mesh is set as in Figure 16(left). Following Remark 30 we obtain $\mu \approx 1200$. We apply the adaptive DS-FE-HMM and observe that the expected convergence rate $\left|p^{0}-p^{H}\right|_{H^{1}(\Omega)} \propto N_{\text {mac }}^{-l / d}$ is obtained as displayed in Figure 13, where $N_{\text {mac }}$ is the number of degrees of freedom of the macro problem. The micro error decays at a faster rate, proportional to $N_{\operatorname{mac}}^{-(l+1) / d}$. This is expected, as we estimate the $L^{2}$-norm by the $H^{1}$-norm in (65). Sample solutions $p^{H}$ with different mesh refinements and the homogenized solution $p^{0}$ are plotted in Figure 14.

For experiments with different FE spaces we use the DS-FE-HMM with periodic BC on micro scale and explicit scale separation, that is, $Y_{\mathrm{F}}^{x, \delta}:=Y_{\mathrm{F}}^{x}$. There, the value of $\delta=$ 


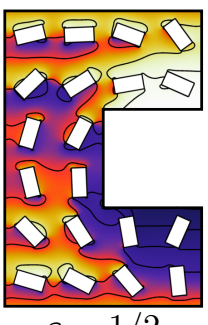

$\varepsilon=1 / 2$

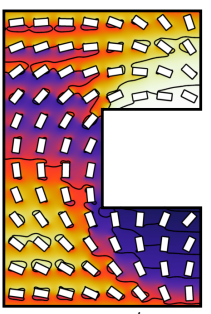

$\varepsilon=1 / 4$

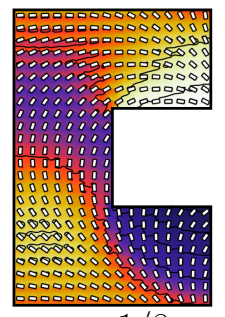

$\varepsilon=1 / 8$

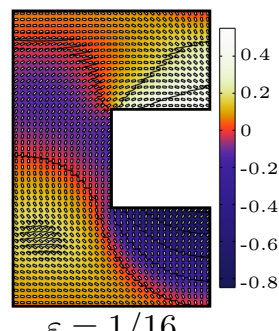

$\varepsilon=1 / 16$

Figure 12: Medium A: Plots of $p^{\varepsilon}$ for different $\varepsilon>0$.
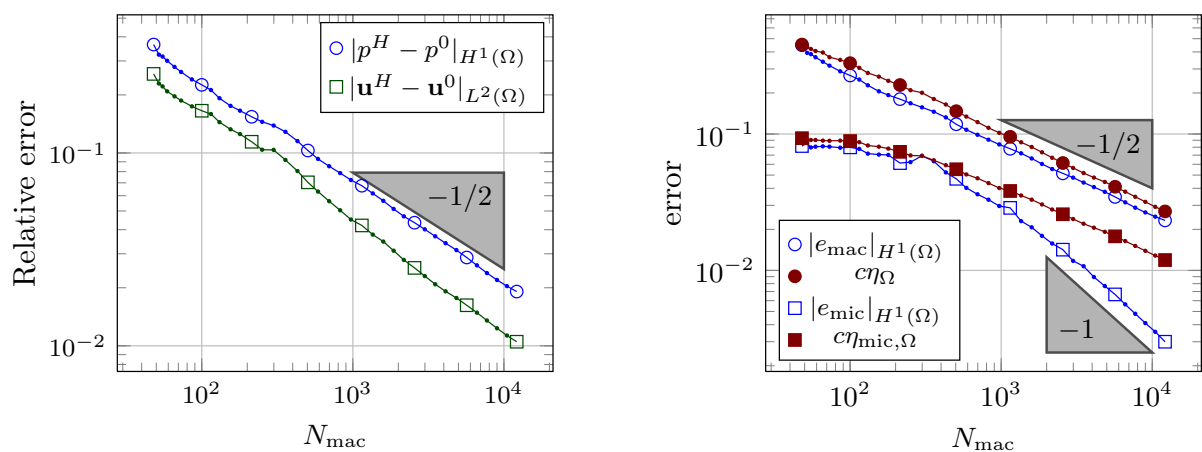

Figure 13: Medium $A$ : Errors analysis $\left(\delta=\varepsilon=10^{-4}\right.$, macro: $\mathbb{P}_{1}$, micro: $\mathbb{P}_{2} / \mathbb{P}_{1}$, periodic BC).
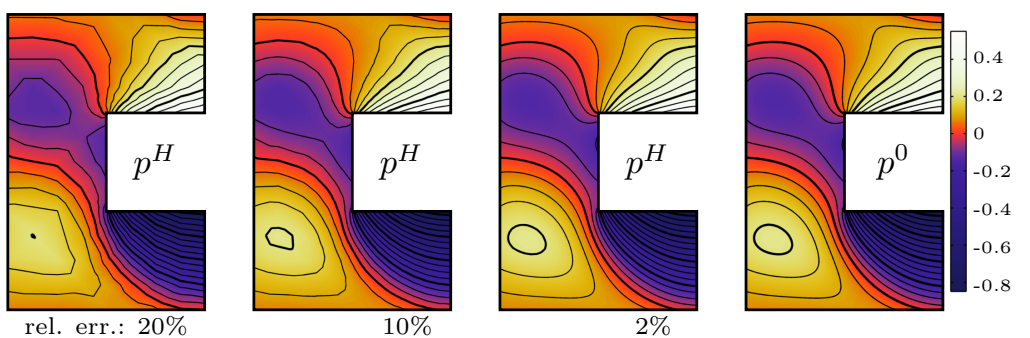

Figure 14: Medium A: $p^{0}$ (right) and DS-FE-HMM solutions $\left(\delta=\varepsilon=10^{-4}\right.$, macro: $\mathbb{P}_{1}$, micro: $\mathbb{P}_{2} / \mathbb{P}_{1}$ and periodic $\mathrm{BC}$ ).

$\varepsilon$ does not affect the computation and the modeling error is thus eliminated. We test six different combinations of micro and macro FEs. The convergence rates displayed in Figure 15 corroborate the theoretical results obtained in Section 5. As in standard adaptive FEM, the
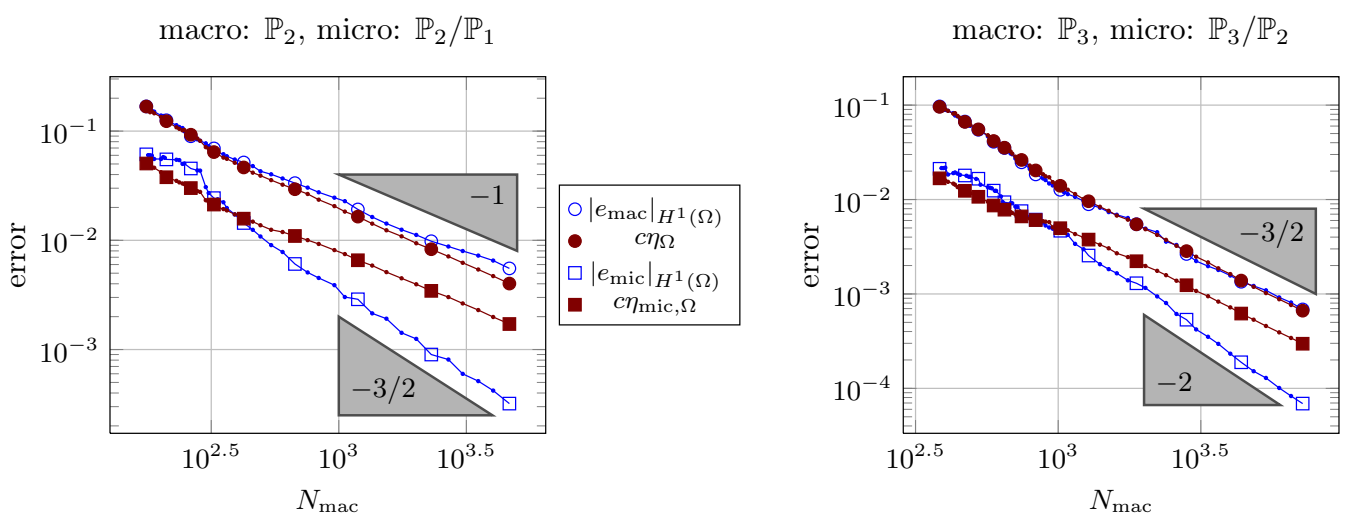

Figure 15: Medium A: Error analysis of DS-FE-HMM with different FE (micro: periodic BC) mesh is more refined close to the corner singularities at points $[1,1]$ and $[1,2]$. With increasing 
$l$, the refinement is even stronger close to the corners. Figure 16 compares meshes for the same relative error of different macro FEs. In Figure 17 we plot the convergence rates versus
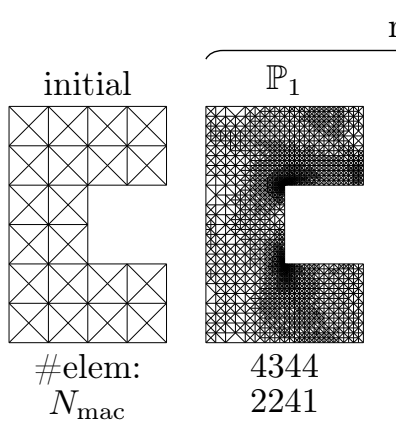
rel. err.: $5 \%$
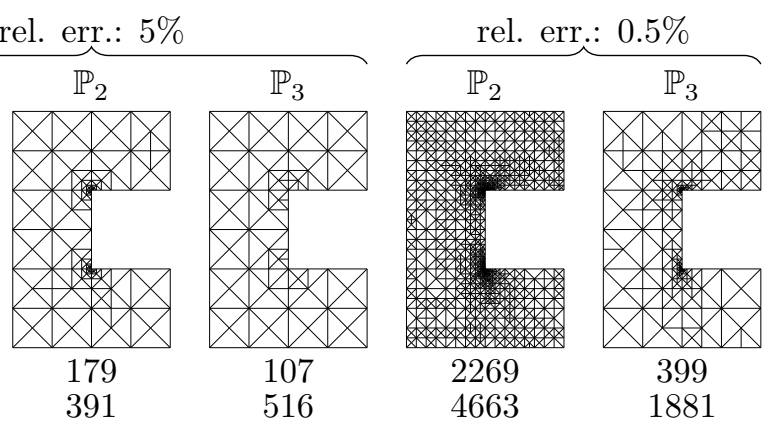

Figure 16: Medium A: Macro meshes at different stages of DS-FE-HMM (micro: periodic BC)

the the total cost of the method, that is, the sum of the degrees of freedom in the macro and all the micro problems. The obtained convergence rate $-\frac{l}{d} \cdot \frac{k+1}{l+k+1}$ is slightly smaller than the optimal convergence rate $-\frac{l}{d} \cdot \frac{k+2}{l+k+2}$. This can be explained because of the suboptimal estimate in (65).

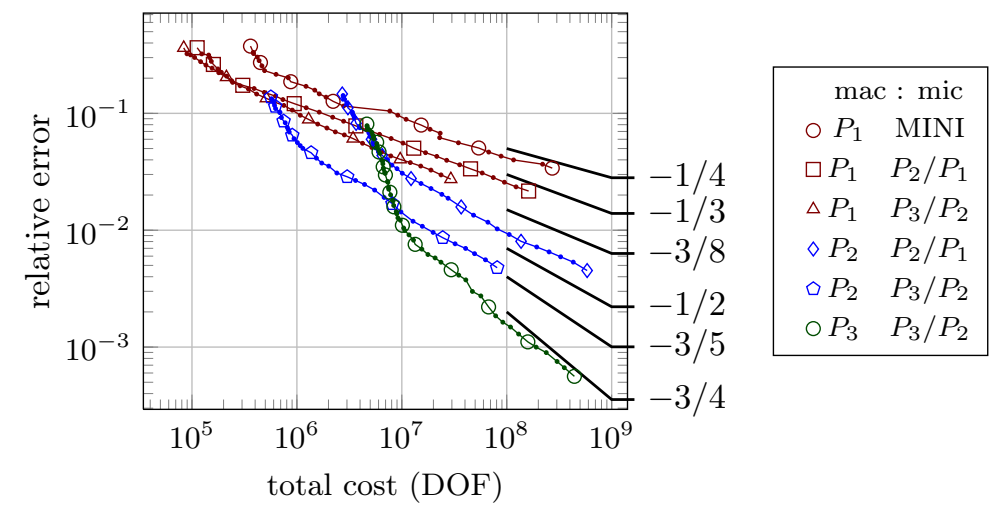

Figure 17: Medium A: Error vs. total cost of DS-FE-HMM (micro: periodic BC)

To show that our method is robust without the precise knowledge of the size of micro domains for the Stokes flow, we changed $\delta$ to be a non-integer multiple of $\varepsilon$ with both Neumann and periodic $\mathrm{BC}$ on the micro problems. The detailed error analysis can be seen in Figure 18.
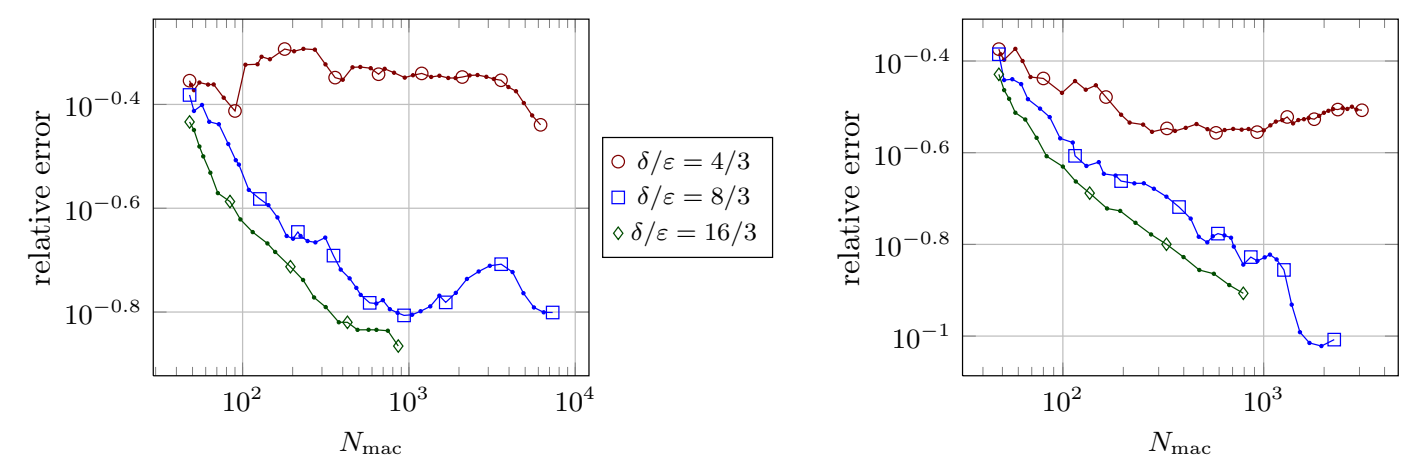

Figure 18: Medium A: Error analysis of DS-FE-HMM with Neumann BC (left) and periodic $\mathrm{BC}$ (right) on micro problems for different $\delta>\varepsilon=10^{-4}$.

7.2. Porous medium $B$. We use the same macro domain $\Omega$ and force filed $\mathbf{f} \equiv$ $\mathbf{f}^{H} \equiv(0,-1)$ as for the porous medium $A$, but the pore geometry is now more involved 
(see Figure 10(c)). The solid part $Y_{\mathrm{S}}^{x}$ consists of three regular hexagons with centers at a distance 0.25 from the point $[0,0]$. The sides of the hexagons $r_{1}, r_{2}, r_{3}$ and the rotation angle $\theta$ are given by $r_{j}(x)=A(\zeta+(j-1) / 3)$ for $j \in\{1,2,3\}$ and $\theta(x)=2 \pi \zeta / 3$, where $\zeta=\left(1+\sin \left(x_{1}\right)\right)\left(1+\sin \left(2 \pi x_{2} / 3\right)\right) / 4$ and $A(\zeta)=0.145+0.035 \sin (2 \pi \zeta)$. The mapping $\varphi$ governing the slow variation of the medium depends on $r_{1}, r_{2}, r_{3}$ and the rotation angle $\theta$. This mapping thus rotates and changes the size of the solid parts.

Porous domains $\Omega_{\varepsilon}$ and fine scale solution $p^{\varepsilon}$ are plotted in Figure 19 for various values of $\varepsilon$. The solutions $p^{\varepsilon}$ were computed as for the medium $A$.

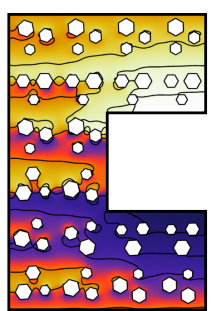

$\varepsilon=1 / 2$

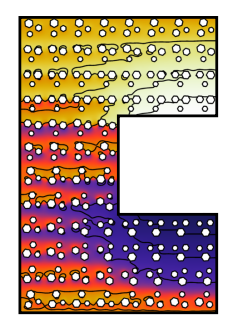

$\varepsilon=1 / 4$

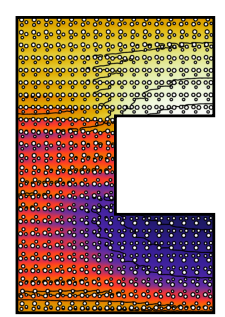

$\varepsilon=1 / 8$

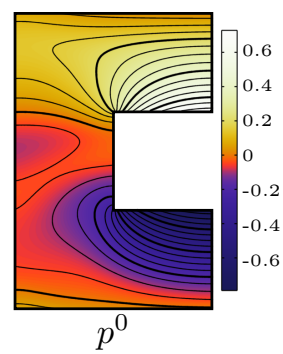

Figure 19: Medium $B: p^{\varepsilon}$ for different $\varepsilon>0$ and the homogenized pressure $p^{0}$.

We performed an experiment with $\mathbb{P}_{1}$ macro $\mathrm{FE}(k=1)$ and $\mathbb{P}_{2} / \mathbb{P}_{1}$ micro $\mathrm{FE}(l=1)$. The convergence rates presented in Figure 21 again corroborate the theoretical results obtained in Section 5 .
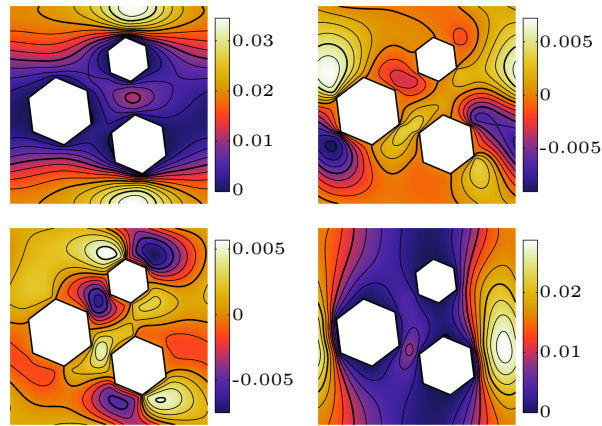

$a^{0}(x) \approx 10^{-3}\left(\begin{array}{cc}9.564 & -0.028 \\ -0.028 & 7.608\end{array}\right)$
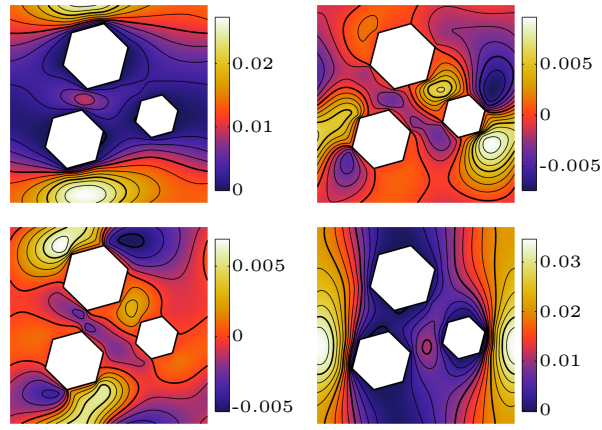

$a^{0}(x) \approx 10^{-3}\left(\begin{array}{cc}6.619 & -0.084 \\ -0.084 & 10.264\end{array}\right)$

Figure 20: Medium $B$ : micro velocity solutions for $x=[0.5,1]$ (left) and $x=[0.5,1.5]$ (right).
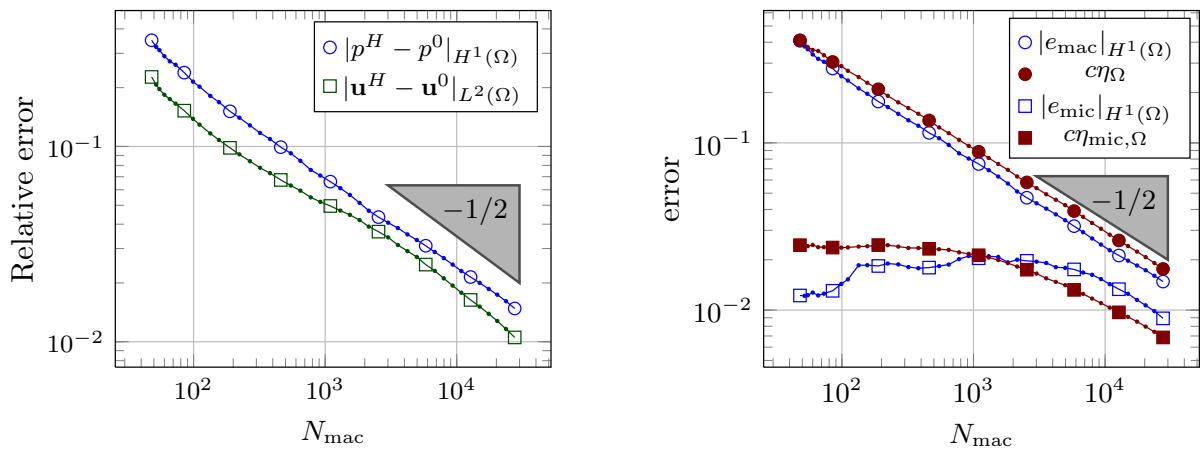

Figure 21: Medium B: DS-FE-HMM error analysis (macro: $\mathbb{P}_{1}$, micro: $\mathbb{P}_{2} / \mathbb{P}_{1}$, periodic BC).

7.3. Porous medium $C$. Let $\Omega$ be a subset of $(0,2) \times(0,2) \times(0,3)$ for which $\left(x_{3}-\right.$ $2)\left(x_{3}-1\right)>0$ or $\max \left(x_{1}, x_{2}\right)<1$ and let $\mathbf{f} \equiv \mathbf{f}^{H} \equiv(0,0,-1)$, see Figure 22 (left). Let the 
faces $(0,2) \times(0,2) \times\{0\}$ and $(0,2) \times(0,2) \times\{3\}$ be periodically connected. We will define a three-dimensional porous structure where the solid part $\Omega \backslash \Omega_{\varepsilon}$ is connected.
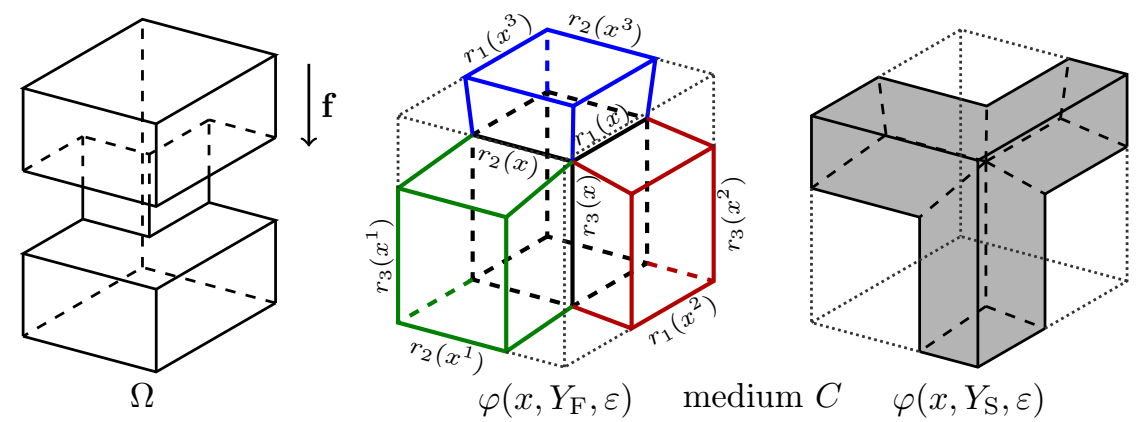

Figure 22: Medium $C$ : macroscopic domain $\Omega$ (left) and a description of the pore structure. The fluid part (middle) consists of a rectangular prism (black) and three connecting polyhedrons (red, green, blue).

We will define the domain $\Omega_{\varepsilon}$ such that it will consist of rectangular prisms spaced in an $\varepsilon$-sized grid, connected in all three basic directions by simple channels (see Figure 24(right)). To describe such medium in the locally periodic fashion, we follow Remark 5 and use the generalized definition of locally periodic porous media, where the map $\varphi(x, y, \varepsilon)$ takes $\varepsilon$ as a parameter. Instead of stating $\varphi$ explicitly, we define $\varphi\left(x, Y_{\mathrm{F}}, \varepsilon\right)$, where $Y_{\mathrm{F}}$ can be set appropriately. While reading the following description, follow Figure 22 (right). We let $\varphi\left(x, Y_{\mathrm{F}}, \varepsilon\right)$ be a rectangular prism of size $r_{1}(x) \times r_{2}(x) \times r_{3}(x)$ located in the corner of $Y$. Its three faces that do not lie on $\partial Y$ are faces of three polyhedrons that reach to the opposite side of $Y$ and these polyhedrons will serve as a connection to the neighboring cells in $\Omega_{\varepsilon}$, which contain rectangular prisms of sizes $r_{1}\left(x^{k}\right) \times r_{2}\left(x^{k}\right) \times r_{3}\left(x^{k}\right)$, where $x^{k}=x+\varepsilon \mathbf{e}^{k}$ for $k \in\{1,2,3\}$. We define $r_{k}(x)=0.5+0.2 \cos (2 \pi(\zeta+(k-1) / 3))$ for $k \in\{1,2,3\}$, where $\zeta(x)=x_{1} / 2-x_{2} / 2+x_{3} / 3$.
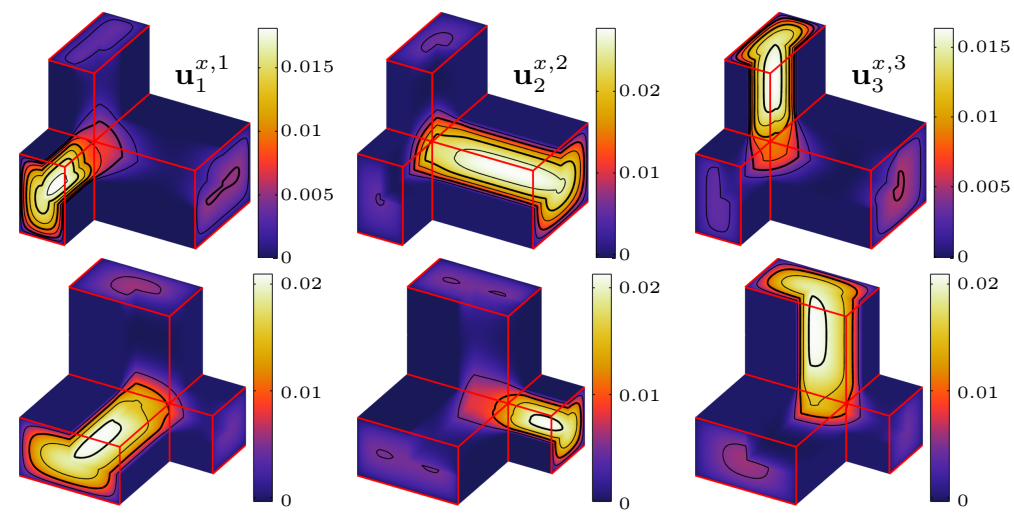

Figure 23: Medium $C$ : an interior view of micro velocity solutions for $x=[0,0,1 / 3]$ with $a^{0}(x) \approx 10^{-3} \operatorname{diag}(1.495,4.638,1.69) \quad\left(\right.$ up) and $x=[0,0,2]$ with $a^{0}(x) \approx$ $10^{-3} \operatorname{diag}(2.895,1.638,2.895)$ (down).

We apply the DS-FE-HMM with $\mathbb{P}_{1}$ macro FEs $(l=1)$, and $\mathbb{P}_{2} / \mathbb{P}_{1}$ micro FEs $(k=1)$. Periodic BC are used on the micro problems and we set $Y_{\mathrm{F}}^{x, \delta}=Y_{\mathrm{F}}^{x}$. Sample micro solutions can be seen in Figure 23. The convergence rates given in Figure 25 are as predicted by the results of Section 5. Despite choosing very coarse initial micro meshes, the macro error dominates the micro error. The adaptive algorithm detects this behavior and allows for coarse micro meshes reducing the computational cost by order of magnitudes compared to a multiscale macro-micro method that would be used with uniform micro mesh refinement.

8. Conclusion. We have presented a multiscale FE method for the Stokes flow in porous media. The method is based on a macroscopic FE discretization of an elliptic problem (Darcy flow) with effective permeability recovered from micro FE solutions of Stokes problems 

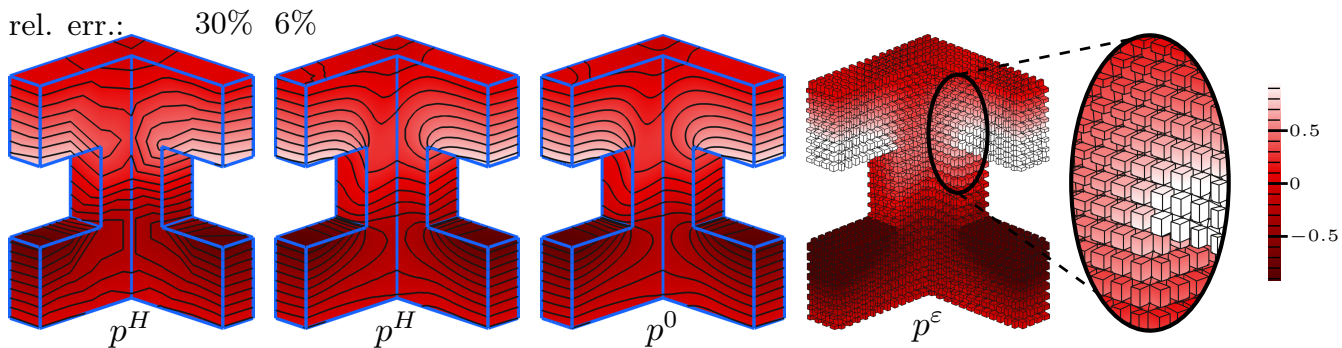

Figure 24: Medium C: DS-FE-HMM solutions with relative errors $30 \%$ and $6 \%, p^{0}$, and $p^{\varepsilon}$ for $\varepsilon=1 / 8$.
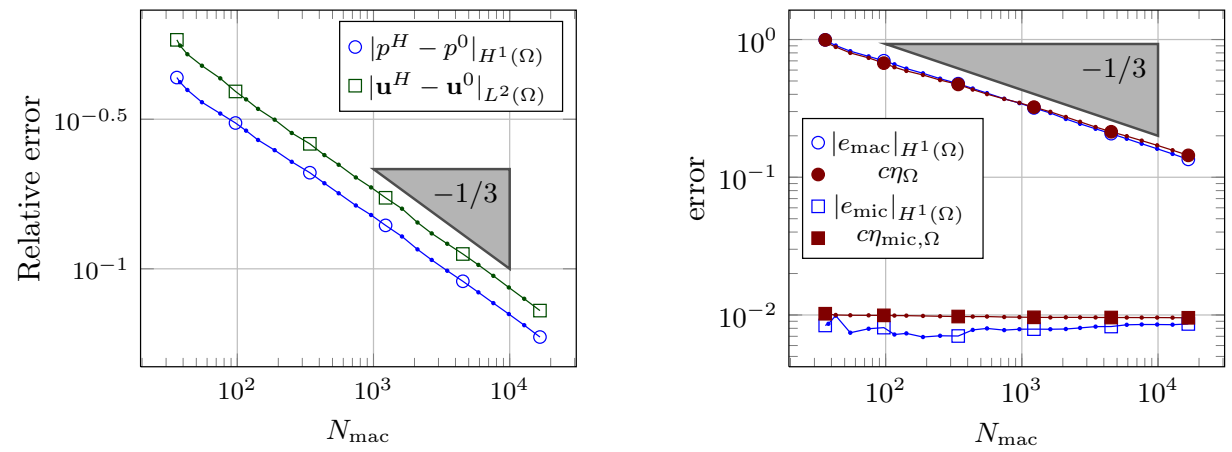

Figure 25: Medium $C$ : DS-FE-HMM error analysis (macro: $\mathbb{P}_{1}$, micro: $\mathbb{P}_{2} / \mathbb{P}_{1}$, periodic BC).

and its computational cost is independent of the pore size. We have focused on a class of problems with non-periodic pore structures that can be obtained from a smooth deformation of a reference pore sampling domain. As the well-posedness of the Darcy problem depends on the Stokes flow at the pore level, we have analyzed classes of microscopic geometries that ensure existence and uniqueness of a solution of the macroscopic problem and its FE discretization. While a priori error analysis has been discussed, our main objective has been to derive an adaptive algorithm combining macroscopic and microscopic mesh refinement. Rigorous a posteriori error estimates have been derived that show efficiency and reliability of the proposed adaptive method as corroborated by numerical experiments for non-periodic two- and three-dimensional problems. The adaptive algorithm presented here also allows for further generalizations and improvements. In particular, the application of goal-oriented adaptive FE methods [39] or reduced basis techniques [3,43] could be used to compute the functionals $\int_{Y_{\mathrm{F}}^{x, \delta}} \mathbf{u}_{j}^{i, x, h}$ and control the error in the quantity of interest $\left\|a^{h}(x)-\bar{a}(x)\right\|_{\mathrm{F}}$.

\section{References.}

[1] A. ABDulLe, On a priori error analysis of fully discrete heterogeneous multiscale FEM, Multiscale Model. Simul., 4 (2005), pp. 447-459.

[2] — A priori and a posteriori error analysis for numerical homogenization: a unified framework, Ser. Contemp. Appl. Math. CAM, 16 (2011), pp. 280-305.

[3] A. Abdulle And Y. BAI, Adaptive reduced basis finite element heterogeneous multiscale method, to appear in Comput. Methods Appl. Mech. Engrg., (2013).

[4] A. Abdulle and A. Nonnenmacher, A posteriori error analysis of the heterogeneous multiscale method for homogenization problems, C. R. Math. Acad. Sci. Paris, 347 (2009), pp. 1081-1086.

[5] — A short and versatile finite element multiscale code for homogenization problem, Comput. Methods Appl. Mech. Engrg, 198 (2009), pp. 2839-2859.

[6] _ Adaptive finite element heterogeneous multiscale method for homogenization problems, Comput. Methods Appl. Mech. Engrg., 200 (2011), pp. 2710-2726. 
[7] — A posteriori error estimate in quantities of interest for the finite element heterogeneous multiscale method, Numer. Methods Partial Differential Equations, 29 (2013), pp. $1441-1800$.

[8] S. Agmon, Lectures on elliptic boundary value problems, Van Nostrand, Princeton, N.J., 1965.

[9] M. Ainsworth And J. T. Oden, A posteriori error estimation in finite element analysis, Comput. Methods Appl. Mech. Engrg., 142 (1997), pp. 1-88.

[10] G. Allaire, Homogenization of the Stokes flow in a connected porous medium, Asymptot. Anal., 2 (1989), pp. 203-222.

[11] _ Homogenization of the Navier-Stokes equations in open sets perforated with tiny holes $i$. abstract framework, a volume distribution of holes, Arch. Ration. Mech. Anal., 113 (1991), pp. 209-259.

[12] S. Alyaev, E. Keilegavlen, and J. M. Nordbotten, Analysis of control volume heterogeneous multiscale methods for single phase flow in porous media, preprint, (2013).

[13] D. N. Arnold, F. Brezzi, And M. Fortin, A stable finite element for the Stokes equations, Calcolo, 21 (1984), pp. 337-344.

[14] D. N. Arnold, A. Mukherjee, And L. Pouly, Locally adapted tetrahedral meshes using bisection, SIAM J. Sci. Comput., 22 (2000), pp. 431-448.

[15] A. Y. Beliaev and S. M. Kozlov, Darcy equation for random porous media, Comm. Pure Appl. Math., 49 (1996), pp. 1-34.

[16] C. Bernardi, Y. Matay, And F. Rapetti, Discrétisations variationnelles de problèmes aux limites elliptiques, vol. 45 of Mathématiques et applications, Springer Verlag, Berlin, 2004.

[17] D. Boffi, F. Brezzi, And M. Fortin, Finite elements for the Stokes problem, in Mixed Finite Elements, Compatibility Conditions, and Applications, Springer, 2008, pp. 45-100.

[18] D. BRAEss, Finite elements: Theory, fast solvers, and applications in solid mechanics, Cambridge University Press, Cambridge, third ed., 2007.

[19] F. Brezzi And M. Fortin, Mixed and hybrid finite element methods, vol. 15 of Springer Series in Computational Mathematics, Springer, 1991.

[20] D. L. Brown, Y. Efendiev, And V. H. HoAng, An efficient hierarchical multiscale finite element method for Stokes equations in slowly varying media, Multiscale Model. Simul., 11 (2013), pp. 30-58.

[21] D. L. Brown, P. Popov, And Y. Efendiev, On homogenization of Stokes flow in slowly varying media with applications to fluid-structure interaction, GEM Int. J. Geomath., 2 (2011), pp. 281-305.

[22] G. A. Chechkin And A. L. Piatnitski, Homogenization of boundary-value problem in a locally periodic perforated domain, Appl. Anal., 71 (1998), pp. 215-235.

[23] L. ChEN AND C.-S. ZHANG, AFEM@Matlab: a matlab package of adaptive finite element methods, tech. rep., Department of Mathematics, University of Maryland at College Park, 2006.

[24] P. G. Ciarlet, The finite element method for elliptic problems, vol. 4 of Studies in Mathematics and its Applications, North-Holland, 1978.

[25] P. ClÉment, Approximation by finite element functions using local regularization, Rev. Fr. Autom. Inform. Rech. Opér., Anal. Numér., 9 (1975), pp. 77-84.

[26] R. Cools, Constructing cubature formulae: the science behind the art, Acta Numer., 6 (1997), pp. 1-54. 
[27] —, An encyclopaedia of cubature formulas, J. Complexity, 19 (2003), pp. 445-453.

[28] H. DARCY, Les fontaines publiques de la ville de Dijon: Exposition et application á suivre et des formules á employer dans les questions de duistribution d'eau, 1856.

[29] M. Dobrowolski, On the LBB condition in the numerical analysis of the Stokes equations, Appl. Numer. Math., 54 (2005), pp. 314-323.

[30] M. S. Espedal, A. Fasano, And A. Mikelić, Filtration in Porous Media and Industrial Application, vol. 1734 of Lecture Notes in Mathematics, Springer, 2000.

[31] G. P. GALDI, An introduction to the mathematical theory of the Navier-Stokes equations: Steady-state problems, Springer Monographs in Mathematics, Springer, second ed., 2011.

[32] C. Geuzaine And J.-F. Remacle, Gmsh: A three-dimensional finite element mesh generator with built-in pre- and post-processing facilities, Internat. J. Numer. Methods Engrg., 79 (2009), pp. 1309-1331.

[33] V. Girault And P.-A. Raviart, Finite Element Methods for Navier-Stokes Equations, Theory and Algorithms, vol. 5 of Springer Series in Computational Mathematics, SpringerVerlag, 1986.

[34] E. Marušić-PAloka AND A. Mikelić, An error estimate for correctors in the homogenization of the Stokes and Navier-Stokes equations in a porous medium, Boll. Unione Mat. Ital., 10 (1996), pp. 661-671.

[35] A. Mikelić, Homogenization theory and applications to filtration through porous media, in Filtration in Porous Media and Industrial Application [30], pp. 127-214.

[36] G. A. Narsilio, O. Buzzi, S. Fityus, T. S. Yun, And D. W. Smith, Upscaling of Navier-Stokes equations in porous media: Theoretical, numerical and experimental approach, Computers and Geotechnics, 36 (2009), pp. 1200-1206.

[37] A. Nonnenmacher, Adaptive Finite Element Methods for Multiscale Partial Differential Equations, vol. No. 5097, École Polytechnique Fédérale de Lausanne, 2011.

[38] Y. Notay, An aggregation-based algebraic multigrid method, Electron. Trans. Numer. Anal., 37 (2010), pp. 123-146.

[39] J. T. Oden, S. Prudhomme, A. Romkes, and P. Bauman, Multiscale modeling of physical phenomena: adaptive control of models, SIAM J. Sci. Comput., 28 (2006), pp. 2359-2389.

[40] M. Ohlberger, A posteriori error estimates for the heterogeneous multiscale finite element method for elliptic homogenization problems, Multiscale Model. Simul., 4 (2005), pp. 88-114.

[41] J. Peters, V. Reichelt, And A. Reusken, Fast iterative solvers for discrete Stokes equations, SIAM J. Sci. Comput., 27 (2005), pp. 646-666.

[42] A. Quarteroni, Numerical Models for Differential Problems, vol. 2 of Modeling, Simulation \& Applications, Springer, 2009.

[43] G. RozzA, D. B. P. Huynh, And A. MAnzoni, Reduced basis approximation and a posteriori error estimation for Stokes flows in parametrized geometries: roles of the infsup stability constants, Numer. Math., 125 (2013), pp. 1-38.

[44] E. SÁnchez-PAlencia, Non-homogeneous media and vibration theory, vol. 127 of Lecture notes in physics, Springer, 1980.

[45] C. Sandström, F. Larsson, K. Runesson, and H. Johansson, A two-scale finite element formulation of Stokes flow in porous media, Comput. Methods Appl. Mech. Engrg., 261-262 (2013), pp. 96-104. 
[46] A. Tamayol and M. Bahrami, Analytical determination of viscous permeability of fibrous porous media, Int. J. Heat Mass Transfer, 52 (2009), pp. 2407-2414.

[47] L. TARTAR, Incompressible fluid flow in a porous medium-convergence of the homogenization process, in Non-homogeneous media and vibration theory [44].

[48] C. TAYLOR AND P. Hood, A numerical solution of the Navier-Stokes equations using the finite element technique, Comput. \& Fluids, 1 (1973), pp. 73-100.

[49] R. Temam, Navier-Stokes equations: Theory and numerical analysis, vol. 343 of AMS Chelsea Publishing, AMS, 1984.

[50] K. VAfaI, Porous Media: Applications in Biological Systems and Biotechnology, CRC Press, 2010.

[51] T. L. van Noorden And A. Muntean, Homogenisation of a locally periodic medium with areas of low and high diffusivity, European J. Appl. Math., 22 (2011), pp. 493-516.

[52] R. VERFÜRTH, Error estimates for a mixed finite element approximation of the Stokes equations, RAIRO. Anal. Numér., 18 (1984), pp. 175-182.

[53] — A posteriori error estimators for the Stokes equations, Numer. Math., 55 (1989), pp. 309-325.

[54] - A review of a posteriori error estimation and adaptive mesh-refinement techniques, Wiley-Teubner, 1996.

[55] B. Verleye, R. Croce, M. Griebel, M. Klitz, S. V. Lomov, G. Morren, H. Sol, I. Verpoest, and D. Roose, Permeability of textile reinforcements: Simulation, influence of shear and validation, Compos. Sci. Technol., 68 (2008), pp. 2804-2810.

[56] M. A. D. Viera, P. N. Sahay, M. Coronado, and A. O. Tapia, Mathematical and Numerical Modeling in Porous Media: Applications in Geosciences, CRC Press, 2012. 